\title{
On $m$-Isometric Semigroups, and 2-Isometric Cogenerators
}

\author{
Eskil Rydheø
}

\begin{abstract}
It is known that a $C_{0}$-semigroup of Hilbert space operators is $m$-isometric if and only if its generator satisfies a certain condition, which we choose to call $m$-skew-symmetry. This paper contains two main results: We provide a Lumer-Phillips type characterization of generators of $m$-isometric semigroups. This is based on the simple observation that $m$-isometric semigroups are quasicontractive. We also characterize cogenerators of 2-isometric semigroups. To this end, our main strategy is to construct a functional model for 2-isometric semigroups with analytic cogenerators. The functional model yields numerous simple examples of non-unitary 2-isometric semigroups, but also allows for the construction of a closed, densely defined, 2-skew-symmetric operator which is not a semigroup generator.
\end{abstract}

Mathematics Subject Classification. Primary 47D06, Secondary 30H99, 47A63, 47B38.

Keywords. Cogenerator, Harmonically weighted Dirichlet space, Infinitesimal generator, Semigroup, 2-isometry, $m$-isometry.

\section{Introduction}

Throughout this paper, we let $\mathcal{H}$ and $\mathcal{E}$ denote complex Hilbert spaces. On their own, these are assumed to be completely general, while together, we will typically let $\mathcal{E}$ denote a certain subspace of $\mathcal{H}$. The word operator always refers to a linear map $A: D(A) \rightarrow \mathcal{H}$, with domain $D(A) \subseteq \mathcal{H}$. While all operators encountered below are closed, we do not insist on this as part of the definition. The space of bounded operators is denoted $\mathcal{L}=\mathcal{L}(\mathcal{H})$. As for operators on $\mathcal{E}$, we are more interested in $\mathcal{L}_{+}=\mathcal{L}_{+}(\mathcal{E})$, the convex cone of positive bounded operators.

This work was supported by the Knut and Alice Wallenberg foundation, scholarship KAW 2016.0442. 
A bounded operator $T$ is called $m$-isometric if

$$
\sum_{k=0}^{m}(-1)^{m-k}\left(\begin{array}{c}
m \\
k
\end{array}\right)\left\|T^{k} x\right\|^{2}=0, \quad x \in \mathcal{H} .
$$

The study of $m$-isometries was initiated by Agler [1], and significantly extended by Agler and Stankus [2-4]. A related notion is that of $m$-symmetric operators, introduced by Helton [10]. By analogy, we say that an operator $A$ is $m$-skew-symmetric if

$$
\sum_{k=0}^{m}\left(\begin{array}{c}
m \\
k
\end{array}\right)\left\langle A^{k} y, A^{m-k} y\right\rangle=0, \quad y \in D\left(A^{m}\right) .
$$

This happens precisely when $i A$ is $m$-symmetric.

If we think of $n \in \mathbb{Z}_{\geq 0}$ as a time variable, then the sequence $\left(T^{n}\right)_{n=0}^{\infty}$ constitutes a semigroup describing the evolution of some abstract linear system in discrete time. It can be shown that if $T$ is an $m$-isometry, then the semigroup $\left(T^{n}\right)_{n=0}^{\infty}$ consists entirely of $m$-isometries. A recent development is the study of $m$-isometric semigroups in continuous time, i.e. $C_{0}$-semigroups of $m$-isometries [5,9]. It is known that a $C_{0}$-semigroup is $m$-isometric if and only if its generator is $m$-skew-symmetric. Moreover, this is equivalent to that, for each $x \in \mathcal{H}$, the function $t \mapsto\left\|T_{t} x\right\|^{2}$ is a polynomial of degree no more than $(m-1)$ [5, Theorem 2.1]. We will prove a slightly more specific result about the function $t \mapsto\left\|T_{t} x\right\|^{2}$ (Theorem 3.3). This readily implies that $m$-isometric semigroups are quasicontractive (Corollary 3.5). Our first main result is then a simple consequence of the Lumer-Phillips theorem:

Theorem 1.1. Let $m \in \mathbb{Z}_{>1}$. An operator $A$ is the generator of an $m$-isometric semigroup $\left(T_{t}\right)_{t \geq 0}$ if and only if the following conditions are satisfied:

(i) $A$ is closed and densely defined.

(ii) There exists $w \geq 0$ such that

$$
\operatorname{Re}\langle A y, y\rangle \leq w\|y\|^{2}, \quad y \in D(A) .
$$

(iii) There exists $\lambda>w$ such that $\lambda-A: D(A) \rightarrow \mathcal{H}$ is surjective.

(iv) $A$ is m-skew-symmetric.

If the above conditions hold, and if $w \geq 0$, then (1.1) is satisfied if and only if $\left(T_{t}\right)_{t \geq 0}$ is quasicontractive with parameter $w$. Moreover, if $\lambda>0$, then $\lambda-A: D(A) \rightarrow \mathcal{H}$ is invertible.

The final assertion of the above theorem follows from the polynomial growth of $\left\|T_{t} x\right\|^{2}$, together with the standard resolvent formula (2.9). Consequently, the spectrum of $A$ lies in the left half of the complex plane. Therefore, $\left(T_{t}\right)_{t \geq 0}$ has a well-defined cogenerator $T \in \mathcal{L}$ given by

$$
T=(A+I)(A-I)^{-1} .
$$

The cogenerator satisfies certain necessary conditions:

Theorem 1.2. Let $m \in \mathbb{Z}_{\geq 1}$. If $\left(T_{t}\right)_{t \geq 0}$ is an m-isometric semigroup, then $\left(T_{t}\right)_{t \geq 0}$ has a well-defined cogenerator $T \in \mathcal{L}$ satisfying the following conditions: 
(i) $T-I$ is injective, and has dense range.

(ii) There exists $w \geq 0$ such that

$$
\|T x\|^{2}-\|x\|^{2} \leq w\|(T-I) x\|^{2}, \quad x \in \mathcal{H} .
$$

(iii) $T$ is m-isometric.

Moreover, (1.2) holds whenever $\left(T_{t}\right)_{t \geq 0}$ is quasicontractive with parameter $w$.

It is classical, e.g. [20, Chapter III, Section 8], that if $T \in \mathcal{L}$ is a contraction, and $T-I$ is injective, then $T$ is the cogenerator of a contractive $C_{0}$-semigroup. Moreover, the semigroup is isometric whenever $T$ is. Note that if $T$ is isometric, then (1.2) is trivially satisfied for any $w \geq 0$. Hence, Theorem 1.2 has a natural converse in the case where $m=1$. We obtain a similar result for $m=2$ :

Theorem 1.3. Let $T \in \mathcal{L}$ be a 2-isometry. Assume further that $1 \notin \sigma_{p}(T)$, and that there exists $w \geq 0$ for which (1.2) is satisfied. Then $T$ is the cogenerator of a $C_{0}$-semigroup $\left(T_{t}\right)_{t \geq 0}$, which is 2-isometric, and quasicontractive with parameter $w$.

Note that Theorem 1.3 does not require $T-I$ to have dense range. This is much like in the isometric case: If $T$ is an isometry, then $T$ and $T^{*}$ have the same invariant vectors, so $T-I$ has dense range if and only if it is injective. Our results imply that $T-I$ has dense range under the more general hypothesis of Theorem 1.3, but the author has not found a direct proof.

The proof of Theorem 1.3 relies primarily on two non-trivial results about 2-isometries, namely a Wold-type decomposition theorem, and a functional model for analytic 2-isometries.

The first of these results (Theorem 2.10) is due to Shimorin [19], and states that any 2-isometry $T$ can be written as a direct sum of a unitary operator, and an analytic 2-isometry. By some classical properties of unitary cogenerators, this allows us to reduce the proof of Theorem 1.3 to the case where $T$ is analytic.

The second result (Theorem 2.11) states that if $T \in \mathcal{L}=\mathcal{L}(\mathcal{H})$ is an analytic 2-isometry, and $\mathcal{E}=\mathcal{H} \ominus T \mathcal{H}$, then $T$ is unitarily equivalent to the operator $M_{z}$, multiplication by the identity function $z \mapsto z$, acting on a harmonically weighted Dirichlet space $\mathcal{D}_{\mu}^{2}(\mathcal{E})$ of $\mathcal{E}$-valued analytic functions on $\mathbb{D}$. The parameter $\mu$ is a measure on the unit circle $\mathbb{T}$, with values in $\mathcal{L}_{+}=\mathcal{L}_{+}(\mathcal{E})$. The correspondence between $T$ and $\mu$ is essentially bijective. Theorem 2.11 was proved by Richter [15] in the case where $\operatorname{dim} \mathcal{E}=1$, and extended to the general case by Olofsson [14]. The main idea behind Theorem 1.3 is that an analytic 2-isometry $T \in \mathcal{L}$ is the cogenerator of a $C_{0}$-semigroup if and only if $M_{z}$ is the cogenerator of a $C_{0}$-semigroup on the corresponding space $\mathcal{D}_{\mu}^{2}(\mathcal{E})$. Such a semigroup is necessarily given by the multiplication operators $\left(M_{\phi_{t}}\right)_{t \geq 0}$, where $\phi_{t}: z \mapsto \exp (t(z+1) /(z-1))$. Our strategy is then to determine all $\mu$ such that the operators $\left(M_{\phi_{t}}\right)_{t \geq 0}$ form a $C_{0}$-semigroup on $\mathcal{D}_{\mu}^{2}(\mathcal{E})$.

After reduction to the analytic case, Theorem 1.3 follows from: 
Theorem 1.4. Let $\mu$ be an $\mathcal{L}_{+}$-valued measure on $\mathbb{T}$. Then the following are equivalent:

(i) For every $t \geq 0, M_{\phi_{t}} \in \mathcal{L}\left(\mathcal{D}_{\mu}^{2}(\mathcal{E})\right)$, and the family $\left(M_{\phi_{t}}\right)_{t \geq 0}$ is a $C_{0^{-}}$ semigroup on $\mathcal{D}_{\mu}^{2}(\mathcal{E})$.

(ii) There exists $w_{1} \geq 0$ such that

$$
\frac{1}{2 \pi} \int_{\mathbb{T}}\langle\mathrm{d} \mu f, f\rangle \leq w_{1}\left\|\left(I-M_{z}\right) f\right\|_{\mathcal{D}_{\mu}^{2}(\mathcal{E})}^{2}, \quad f \in \mathcal{P}_{a}(\mathcal{E}) .
$$

(iii) The set function $\tilde{\mu}: E \mapsto \frac{1}{2 \pi} \int_{E} \frac{\mathrm{d} \mu(\zeta)}{|1-\zeta|^{2}}$ is an $\mathcal{L}_{+}$-valued measure, and there exists $w_{2} \geq 0$ such that

$$
\frac{1}{2 \pi} \int_{\mathbb{T}}\langle\mathrm{d} \tilde{\mu} f, f\rangle \leq w_{2}\|f\|_{\mathcal{D}_{\mu}^{2}(\mathcal{E})}^{2}, \quad f \in \mathcal{P}_{a}(\mathcal{E}) .
$$

If either of the above conditions is satisfied, then the semigroup $\left(M_{\phi_{t}}\right)_{t \geq 0}$ is 2isometric, has cogenerator $M_{z}$, and is quasicontractive with some parameter $w \geq 0$. The optimal (smallest possible) values for $w, w_{1}$ and $w_{2}$ coincide.

Remark 1.5. For an $\mathcal{L}_{+}$-valued measure $\mu$, and $x \in \mathcal{E}$, the set function $\mu_{x, x}$ : $E \mapsto\langle\mu(E) x, x\rangle$ defines a finite positive Borel measure. If the inequality (1.4) holds for constant functions, then

$$
\frac{1}{2 \pi} \int_{\mathbb{T}} \frac{\mathrm{d} \mu_{x, x}(\zeta)}{|1-\zeta|^{2}} \leq w_{2}\|x\|^{2}, \quad x \in \mathcal{E} .
$$

On the other hand, if $\mu$ is any measure satisfying the above inequality, then $E \mapsto \int_{E} \frac{\mathrm{d} \mu(\zeta)}{|1-\zeta|^{2}}$ defines an $\mathcal{L}_{+}$-valued measure. Hence, when attempting to verify condition (iii) of Theorem 1.4, the inequality (1.5) is a natural first step.

Any bounded operator $A$ generates an invertible $C_{0}$-semigroup $\left(e^{t A}\right)_{t \geq 0}$. As a special case of [5, Theorem 2.1] (or Theorem 1.1), stated explicitly as [5, Corollary 2.3], the semigroup is $m$-isometric if and only if $A$ is $m$-skewsymmetric. A limitation of this conclusion is that if $m$ is even, then by [2, Proposition 1.23], $\left(e^{t A}\right)_{t \geq 0}$ is in fact $(m-1)$-isometric. In particular, any 2 -isometric $C_{0}$-semigroup with bounded generator is unitary. On the other hand, Theorem 1.4 allows one to produce numerous examples of non-unitary 2-isometric semigroups. In particular, such semigroups exist. One may also use Theorem 1.4 to construct a closed, densely defined, 2-skew-symmetric operator $A$, with the property that $\lambda-A: D(A) \rightarrow \mathcal{H}$ is surjective for any $\lambda>0$, but which is not the generator of a $C_{0}$-semigroup. This shows that the conditions (1.1) and (1.2) are not superfluous.

The paper is organized as follows: In Sect. 2 we introduce some notation and preliminary material. In Sect. 3 we discuss $m$-isometric semigroups. In particular, we prove Theorems 1.1, and 1.2. In Sect. 4 we prove Theorems 1.3, and 1.4. In Sect. 5 we discuss some examples related to Theorem 1.4. In Sect. 6 we briefly mention the wider context of $m$-concave semigroups. 


\section{Notation and Preliminaries}

We use the notation $\mathbb{D}:=\{z \in \mathbb{C} ;|z|<1\}$ for the open unit disc, and $\mathbb{T}:=\{z \in \mathbb{C} ;|z|=1\}$ for the unit circle of the complex plane $\mathbb{C}$. By $\lambda$ we denote Lebesgue (arc length) measure on $\mathbb{T}$, while $\mathrm{d} A$ will signify integration with respect to area measure on $\mathbb{C}$. We also use $\partial \Omega$ and $\bar{\Omega}$ to denote the boundary and closure of $\Omega \subset \mathbb{C}$, respectively.

Given integers $m \geq k \geq 0$, we let $\left(\begin{array}{c}m \\ k\end{array}\right)=\frac{m !}{k !(m-k) !}$ denote the standard binomial coefficients. If the integers $m$ and $k$ do not satisfy the prescribed inequalities, then we set $\left(\begin{array}{c}m \\ k\end{array}\right)=0$. With this convention, the well-known relation

$$
\left(\begin{array}{c}
m \\
k
\end{array}\right)=\left(\begin{array}{c}
m-1 \\
k-1
\end{array}\right)+\left(\begin{array}{c}
m-1 \\
k
\end{array}\right)
$$

is valid whenever $m \geq 1$ and $k \in \mathbb{Z}$. This will be used repeatedly.

Given an operator $A$, we let $\sigma(A), \sigma_{p}(A), \sigma_{a p}(A)$, and $W(A)$ respectively denote the spectrum, point spectrum, approximate point spectrum, and numerical range of $A$, i.e.

$$
\begin{aligned}
\sigma(A) & :=\{z \in \mathbb{C} ; z-A: D(A) \rightarrow \mathcal{H} \text { is not bijective }\}, \\
\sigma_{p}(A) & :=\{z \in \mathbb{C} ; z-A: D(A) \rightarrow \mathcal{H} \text { is not injective }\}, \\
\sigma_{a p}(A) & :=\{z \in \mathbb{C} ; z-A: D(A) \rightarrow \mathcal{H} \text { is not bounded below }\}, \\
W(A) & :=\left\{\langle A y, y\rangle_{\mathcal{H}} \in \mathbb{C} ; y \in D(A),\|y\|=1\right\} .
\end{aligned}
$$

We also let $\rho(A):=\mathbb{C} \backslash \sigma(A)$. If $z \in \rho(A)$, and $A$ is closed, then $(z-A)^{-1}$ : $\mathcal{H} \rightarrow D(A)$ is bounded by the closed graph theorem. On the other hand, if $(z-A)^{-1}$ is bounded, then $A$ is closed.

Given a family $\left\{S_{i}\right\}_{i \in I}$ of subsets of $\mathcal{H}$, we let $\bigvee_{i \in I} S_{i}$ denote the smallest closed subspace of $\mathcal{H}$ that contains each $S_{i}$.

\section{1. $m$-Isometries, and $m$-Skew-Symmetries}

Let $m \in \mathbb{Z}_{\geq 0}, T \in \mathcal{L}$, and define

$$
\beta_{m}(T)=\sum_{j=0}^{m}(-1)^{m-j}\left(\begin{array}{c}
m \\
j
\end{array}\right) T^{* j} T^{j} .
$$

Apart from a normalizing factor, this agrees with the notation from [2]. A straightforward consequence of (2.1) is that

$$
\beta_{m+1}(T)=T^{*} \beta_{m}(T) T-\beta_{m}(T) .
$$

We also have the following formula, valid for $k \in \mathbb{Z}_{\geq 0}, T \in \mathcal{L}$ :

$$
T^{* k} T^{k}=\sum_{j=0}^{\infty}\left(\begin{array}{l}
k \\
j
\end{array}\right) \beta_{j}(T) .
$$

Note that with our notational convention for binomial coefficients, the above right-hand side has at most $k+1$ non-zero terms. 
If $\beta_{m}(T)=0$, then we say that $T$ is an m-isometry. By (2.2), any such operator is also an $(m+1)$-isometry. Moreover, (2.3) implies

$$
\left\|T^{k} x\right\|^{2}=\sum_{j=0}^{m-1}\left(\begin{array}{l}
k \\
j
\end{array}\right)\left\langle\beta_{j}(T) x, x\right\rangle, \quad x \in \mathcal{H} .
$$

Thus, $\left\|T^{k} x\right\|^{2}$ is polynomial in $k$ whenever $x \in \mathcal{H}$, and $\left\|T^{k}\right\|^{2} \lesssim(1+k)^{m-1}$. Gelfand's formula for the spectral radius yields that $\sigma(T) \subseteq \overline{\mathbb{D}}$. A more careful analysis reveals that $\sigma_{a p}(T) \subseteq \mathbb{T}$, see [2, Lemma 1.21]. By the general fact that $\partial \sigma(T) \subseteq \sigma_{a p}(T)$, it follows that an $m$-isometry is either invertible, in which case $\sigma(T) \subseteq \mathbb{T}$, or it is not invertible, in which case $\sigma(T)=\overline{\mathbb{D}}$.

Given $m \in \mathbb{Z}_{\geq 1}$, and an operator $A$, we define the sesquilinear form $\alpha_{m}^{A}$ by

$$
\alpha_{m}^{A}\left(y_{1}, y_{2}\right)=\sum_{j=0}^{m}\left(\begin{array}{c}
m \\
j
\end{array}\right)\left\langle A^{j} y_{1}, A^{m-j} y_{2}\right\rangle, \quad y_{1}, y_{2} \in D\left(A^{m}\right) .
$$

It will be convenient to write $\alpha_{m}^{A}(y)$ instead of $\alpha_{m}^{A}(y, y)$. By (2.1),

$$
\alpha_{m+1}^{A}\left(y_{1}, y_{2}\right)=\alpha_{m}^{A}\left(A y_{1}, y_{2}\right)+\alpha_{m}^{A}\left(y_{1}, A y_{2}\right) \text {. }
$$

We say that $A$ is $m$-skew-symmetric if $\alpha_{m}^{A}$ vanishes identically.

The relation between $m$-isometries and $m$-skew-symmetries has been frequently exploited in previous works $[5,11]$. For easy reference, we state and prove the following result, which is implicit in $[17$, p. 425]:

Lemma 2.1. Let $A$ be a closed operator, with $1 \in \rho(A)$, and define $T \in \mathcal{L}$ by

$$
T=(A+I)(A-I)^{-1} \text {. }
$$

Then $T-I$ is injective, $D(A)=(T-I) \mathcal{H}$, and $A=(T+I)(T-I)^{-1}$. Moreover, for $m \in \mathbb{Z}_{\geq 0}$, and $x_{1}, x_{2} \in \mathcal{H}$,

$$
\left\langle\beta_{m}(T) x_{1}, x_{2}\right\rangle=2^{m} \alpha_{m}^{A}\left((A-I)^{-m} x_{1},(A-I)^{-m} x_{2}\right) .
$$

Proof. Since $A$ is closed, $(A-I)^{-1}: \mathcal{H} \rightarrow D(A)$ is bounded. It is easy to see that $T=I+2(A-I)^{-1}$. Hence, the bounded operator $T-I$ is injective, with left-inverse $\frac{1}{2}(A-I)$. Moreover, $D(A)=(T-I) \mathcal{H}$. The fact that $A=(T+I)(T-I)^{-1}$ is a simple algebraic verification. see that

It is clear that (2.7) holds for $m=0$. For a general $m$, we use (2.2) to

$$
\begin{aligned}
& \left\langle\beta_{m}(T)(T+I) x_{1},(T-I) x_{2}\right\rangle \\
& \quad=\left\langle\beta_{m+1}(T) x_{1}, x_{2}\right\rangle-\left\langle\beta_{m}(T) T x_{1}, x_{2}\right\rangle+\left\langle\beta_{m}(T) x_{1}, T x_{2}\right\rangle
\end{aligned}
$$

Since $\beta_{m}(T)^{*}=\beta_{m}(T)$, the above identity, with $x_{1}$ and $x_{2}$ interchanged, implies

$$
\begin{aligned}
& \left\langle\beta_{m}(T)(T-I) x_{1},(T+I) x_{2}\right\rangle \\
& \quad=\left\langle\beta_{m+1}(T) x_{1}, x_{2}\right\rangle+\left\langle\beta_{m}(T) T x_{1}, x_{2}\right\rangle-\left\langle\beta_{m}(T) x_{1}, T x_{2}\right\rangle
\end{aligned}
$$


Adding these two identities,

$$
\begin{aligned}
& 2\left\langle\beta_{m+1}(T) x_{1}, x_{2}\right\rangle \\
& \quad=\left\langle\beta_{m}(T)(T+I) x_{1},(T-I) x_{2}\right\rangle+\left\langle\beta_{m}(T)(T-I) x_{1},(T+I) x_{2}\right\rangle .
\end{aligned}
$$

Since $T+I=2 A(A-I)^{-1}$, and $T-I=2(A-I)^{-1}$, we therefore have

$$
\begin{aligned}
& \left\langle\beta_{m+1}(T) x_{1}, x_{2}\right\rangle=2\left\langle\beta_{m}(T) A(A-I)^{-1} x_{1},(A-I)^{-1} x_{2}\right\rangle \\
& \quad+2\left\langle\beta_{m}(T)(A-I)^{-1} x_{1}, A(A-I)^{-1} x_{2}\right\rangle .
\end{aligned}
$$

Assuming that (2.7) holds, (2.6) implies

$$
\left\langle\beta_{m+1}(T) x_{1}, x_{2}\right\rangle=2^{m+1} \alpha_{m+1}^{A}\left((A-I)^{-m-1} x_{1},(A-I)^{-m-1} x_{2}\right) .
$$

Hence, we obtain (2.7) by induction over $m$.

\section{2. $C_{0}$-Semigroups}

By a semigroup we mean a one-parameter family $\left(T_{t}\right)_{t \geq 0} \subset \mathcal{L}$, such that $T_{0}=I$, and $T_{s+t}=T_{s} T_{t}$ for $s, t \geq 0$. For a detailed treatment of the facts outlined below, we refer to [8, Chapter II].

A semigroup is called a $C_{0}$-semigroup, or strongly continuous, if for every $x \in \mathcal{H}$ the orbit map $\xi_{x}:[0, \infty) \ni t \mapsto T_{t} x \in \mathcal{H}$ is continuous. Given a $C_{0^{-}}$ semigroup $\left(T_{t}\right)_{t \geq 0}$, the uniform boundedness principle implies that $\left(T_{t}\right)_{0 \leq t \leq 1}$ is a bounded family in $\mathcal{L}$. The semigroup property then implies that $\left(T_{t}\right)_{t \geq 0}$ is exponentially bounded, i.e. there exists $M \geq 1$ and $w \in \mathbb{R}$ such that

$$
\left\|T_{t}\right\|_{\mathcal{L}} \leq M e^{w t}, \quad t \geq 0 .
$$

If (2.8) holds with $M=1$, then we say that $\left(T_{t}\right)_{t \geq 0}$ is quasicontractive with parameter $w$. A quasicontractive semigroup with parameter 0 is simply called contractive.

The (infinitesimal) generator of $\left(T_{t}\right)_{t \geq 0}$ is the operator $A$ defined by

$$
A y=\lim _{t \rightarrow 0^{+}} \frac{T_{t} y-y}{t} .
$$

Its domain $D(A)$ is the subspace of $y \in \mathcal{H}$ such that the above limit exists. The generator of a $C_{0}$-semigroup is closed, densely defined, and uniquely determines the semigroup. If $y \in D(A)$ and $t \geq 0$, then $T_{t} y \in D(A)$, and $A T_{t} y=T_{t} A y$.

Let $x \in \mathcal{H}$, and $\lambda>w$, where $w$ is as in (2.8). Since $t \mapsto T_{t} x$ is continuous, the integral $\int_{0}^{\infty} T_{t} x e^{-\lambda t} \mathrm{~d} t$ is well-defined as a generalized Riemann integral. It is easy to show that in this sense,

$$
(\lambda-A)^{-1}=\int_{0}^{\infty} T_{t} e^{-\lambda t} \mathrm{~d} t .
$$

Not every closed, densely defined operator is the generator of a $C_{0^{-}}$ semigroups. A fundamental result in this direction is the so-called LumerPhillips theorem:

Theorem 2.2. Let $w \geq 0$, and $A$ be an operator. Then $A$ is the generator of $a$ quasicontractive $C_{0}$-semigroup with parameter $w$ if and only if the following conditions hold:

(i) A is closed and densely defined. 
(ii) $A$ is w-dissipative, i.e.

$$
\operatorname{Re}\langle A y, y\rangle \leq w\|y\|^{2}, \quad y \in D(A) .
$$

(iii) There exists $\lambda>w$ such that $\lambda-A: D(A) \rightarrow \mathcal{H}$ is surjective.

Remark 2.3. The above theorem is typically stated for $w=0$. The general version follows if we consider the operator $A-w$, and the corresponding semigroup $\left(e^{-w t} T_{t}\right)_{t \geq 0}$.

An elementary calculation using the inner product shows that $A$ is 0 dissipative if and only if

$$
\|(\lambda-A) y\| \geq \lambda\|y\|, \quad y \in D(A), \lambda>0 .
$$

Consequently, $\lambda-A$ is injective in this case. Moreover, the above inequality turns out to be the appropriate analogue of (ii) when studying semigroups of operators on Banach spaces.

If the above conditions (i)-(iii) hold, then $\lambda-A: D(A) \rightarrow \mathcal{H}$ is in fact surjective for any $\lambda>w$.

\subsection{Operator Measures}

Let $\mathfrak{S}$ denote the Borel $\sigma$-algebra of subsets of $\mathbb{T}$. An $\mathcal{L}_{+}$-valued measure is a finitely additive set function $\mu: \mathfrak{S} \rightarrow \mathcal{L}_{+}$with the property that for every $x, y \in \mathcal{E}$, the set function $\mu_{x, y}: E \mapsto\langle\mu(E) x, y\rangle$ defines a complex regular Borel measure. For each $E \in \mathfrak{S}$, it holds that

$$
\left|\mu_{x, y}\right|(E) \leq \mu_{x, x}(E)^{1 / 2} \mu_{y, y}(E)^{1 / 2} .
$$

We refer to the proof of [13, Proposition 1.1].

Given a bounded (Borel) measurable function $f: \mathbb{T} \rightarrow \mathbb{C}$, we can define the sesquilinear form $J_{f}:(x, y) \mapsto \int_{\mathbb{T}} f \mathrm{~d} \mu_{x, y}$. It follows from $(2.10)$, that

$$
\left|J_{f}(x, y)\right| \leq\|f\|_{\infty}\|\mu(\mathbb{T})\|\|x\|\|y\| .
$$

By standard functional analytic considerations, the above inequality implies the existence of a uniquely determined operator $I_{f} \in \mathcal{L}$ such that $\left\langle I_{f} x, y\right\rangle=$ $\int_{\mathbb{T}} f \mathrm{~d} \mu_{x, y}$. We denote the operator $I_{f}$ by $\int_{\mathbb{T}} f \mathrm{~d} \mu$. The integral thus defined satisfies the triangle type inequality

$$
\left\|\int_{\mathbb{T}} f \mathrm{~d} \mu\right\| \leq\|f\|_{\infty}\|\mu(\mathbb{T})\|
$$

We will need the following version of the Cauchy-Schwarz inequality:

Lemma 2.4. Let $\mu$ be an $\mathcal{L}_{+}$-valued measure. If $x, y \in \mathcal{E}$, and $f, g: \mathbb{T} \rightarrow \mathbb{C}$ are Borel measurable functions, then

$$
\int_{\mathbb{T}}|f g| \mathrm{d}\left|\mu_{x, y}\right| \leq\left(\int_{\mathbb{T}}|f|^{2} \mathrm{~d} \mu_{x, x}\right)^{1 / 2}\left(\int_{\mathbb{T}}|g|^{2} \mathrm{~d} \mu_{y, y}\right)^{1 / 2} .
$$

Proof. When $f$ and $g$ are simple functions, (2.11) follows from (2.10), and the Cauchy-Schwarz inequality for finite sums. General functions are approximated in the standard fashion. 
Two instances of the above integral will be particularly interesting to us, namely the Fourier coefficients

$$
\hat{\mu}(n)=\frac{1}{2 \pi} \int_{\mathbb{T}} \overline{\zeta^{n}} \mathrm{~d} \mu(\zeta), \quad n \in \mathbb{Z},
$$

and the Poisson extension

$$
P_{\mu}(z)=\frac{1}{2 \pi} \int_{\mathbb{T}} \frac{1-|z|^{2}}{|\zeta-z|^{2}} \mathrm{~d} \mu(\zeta), \quad z \in \mathbb{D} .
$$

If we let $r=|z|$, then

$$
\frac{1-|z|^{2}}{|\zeta-z|^{2}}=\sum_{n \in \mathbb{Z}} r^{|n|}\left(\frac{z}{r}\right)^{n} \overline{\zeta^{n}} .
$$

By the Weierstrass test, this series converges uniformly in $\zeta$. Using term by term integration, we conclude that

$$
P_{\mu}(z)=\sum_{n \in \mathbb{Z}} \hat{\mu}(n) r^{|n|}\left(\frac{z}{r}\right)^{n} .
$$

With the above construction, the integral $\int f \mathrm{~d} \mu$ is only defined when $f$ is bounded. As a remedy for this, adequate for our purposes, we use the following construction: Let $\mu$ be an $\mathcal{L}_{+}$-valued measure, and $h$ a scalar-valued function. If there exists $C>0$ such that

$$
\int_{\mathbb{T}}|h| \mathrm{d} \mu_{x, x} \leq C\|x\|^{2}, \quad x \in \mathcal{E},
$$

then one can define a new set function $\mu_{h}$ by

$$
\left\langle\mu_{h}(E) x, y\right\rangle=\int_{E} h \mathrm{~d} \mu_{x, y} .
$$

By Lemma 2.4, the above right-hand side has modulus less than

$$
\left(\int_{\mathbb{T}}|h| \mathrm{d} \mu_{x, x}\right)^{1 / 2}\left(\int_{\mathbb{T}}|h| \mathrm{d} \mu_{y, y}\right)^{1 / 2} \leq C\|x\|\|y\| .
$$

This estimate implies that $\mu_{h}$ is another $\mathcal{L}_{+}$-valued measure. If $f$ is bounded, then we may take $\int f \mathrm{~d} \mu_{h}$ as a definition of $\int f h \mathrm{~d} \mu$.

Remark 2.5. We will only use the above construction of $\int f h \mathrm{~d} \mu$ in the setting where $h$ is a fixed function. However, the following may be of independent interest: If $f_{1} h_{1}=f_{2} h_{2}$, and the measures $\mu_{i}=\mu_{h_{i}}$ are defined as above, then

$$
\left\langle\int f_{i} \mathrm{~d} \mu_{i} x, y\right\rangle=\int f_{i} \mathrm{~d}\left(\mu_{i}\right)_{x, y}=\int f_{i} h_{i} \mathrm{~d} \mu_{x, y} .
$$

Hence,

$$
\int f_{1} h_{1} \mathrm{~d} \mu=\int f_{2} h_{2} \mathrm{~d} \mu \text {. }
$$


Let $f, g: \mathbb{T} \rightarrow \mathcal{E}$ be continuous functions, and identify these with their respective Poisson extensions. For $0<r<1$ and $\zeta \in \mathbb{T}$,

$$
\left\langle P_{\mu}(r \zeta) f(r \zeta), g(r \zeta)\right\rangle=\sum_{k, l, n \in \mathbb{Z}}\langle\hat{\mu}(n) \hat{f}(k), \hat{g}(l)\rangle r^{|n|+|k|+|l|} \zeta^{n+k-l},
$$

and the right-hand side converges uniformly in $\zeta$. Integrating with respect to $\mathrm{d} \lambda(\zeta)$ yields

$$
\int_{\mathbb{T}}\left\langle P_{\mu}(r \zeta) f(r \zeta), g(r \zeta)\right\rangle \mathrm{d} \lambda(\zeta)=2 \pi \sum_{k, l \in \mathbb{Z}} r^{|k|+|l|+|k-l|}\langle\hat{\mu}(l-k) \hat{f}(k), \hat{g}(l)\rangle .
$$

This motivates us to define $\int\langle\mathrm{d} \mu \cdot, \cdot\rangle$ by

$$
\frac{1}{2 \pi} \int_{\mathbb{T}}\langle\mathrm{d} \mu f, g\rangle=\sum_{k, l \in \mathbb{Z}}\langle\hat{\mu}(l-k) \hat{f}(k), \hat{g}(l)\rangle,
$$

provided that the above right-hand side is absolutely convergent.

It seems clear that any reasonable definition of $\int\langle\mathrm{d} \mu \cdot, \cdot\rangle$ should satisfy (2.12). On the other hand, it is a bit awkward to require so much regularity for a function to be square integrable. The next example is a digression from the primary topic of this paper, but may still be of interest.

Example. For $k \in \mathbb{Z}_{\geq 1}$, let $I_{k}$ denote the arc $\left\{e^{i t} ; t \in\left[2^{-k}, 2^{1-k}\right)\right\} \subset \mathbb{T}$, and define the set function $\mu$ by

$$
\langle\mu(E) x, y\rangle=\sum_{k=1}^{\infty} 2^{k} \lambda\left(E \cap I_{k}\right)\left\langle x, e_{k}\right\rangle\left\langle e_{k}, y\right\rangle, \quad x, y \in \mathcal{E},
$$

where $\left(e_{k}\right)_{k=1}^{\infty}$ is some orthonormal sequence in $\mathcal{E}$. Then $\mu$ is an $\mathcal{L}_{+}$-valued measure. For a simple function $f=\sum_{n} x_{n} \mathbb{1}_{E_{n}}$, it might seem reasonable to define $\int\langle\mathrm{d} \mu f, f\rangle$ as the finite sum

$$
\sum_{n}\left\langle\mu\left(E_{n}\right) x_{n}, x_{n}\right\rangle
$$

However, if $f_{N}=\sum_{k=1}^{N} e_{k} \mathbb{1}_{I_{k}}$, then the above sum is equal to $N$, even though $\left\|f_{N}\right\|_{L^{\infty}}=1$. This may help explain why we have chosen (2.12) as our definition of $\int\langle\mathrm{d} \mu \cdot, \cdot\rangle$.

\subsection{Function Spaces}

The space of analytic polynomials $\sum_{k=0}^{N} a_{k} z^{k}$ with coefficients $a_{k} \in \mathcal{E}$ is denoted by $\mathcal{P}_{a}(\mathcal{E})$. As a notational convention, we write $\mathcal{P}_{a}$ in place of $\mathcal{P}_{a}(\mathbb{C})$. The same principle applies to all function spaces described below.

Let $f$ be a function which is analytic in a neighbourhood of the origin. The $k$ th Maclaurin coefficient of $f$ is denoted by $\hat{f}(k)$. By $\mathcal{D}_{a}(\mathcal{E})$, we denote the space of $\mathcal{E}$-valued analytic functions whose Maclaurin coefficients $(\hat{f}(k))_{k=0}^{\infty}$ decay faster than any power of $k$. These are precisely the analytic functions on $\mathbb{D}$ which extend to smooth functions on $\overline{\mathbb{D}}$.

The Hardy space $H^{2}(\mathcal{E})$ consists of all functions $f: z \mapsto \sum_{k=0}^{\infty} \hat{f}(k) z^{k}$, where $\hat{f}(k) \in \mathcal{E}$, and $\|f\|_{H^{2}(\mathcal{E})}^{2}:=\sum_{k=0}^{\infty}\|\hat{f}(k)\|_{\mathcal{E}}^{2}<\infty$. Using a standard radius of convergence formula, functions in $H^{2}(\mathcal{E})$ are seen to be analytic on 
$\mathbb{D}$. The subspaces $\mathcal{P}_{a}(\mathcal{E})$ and $\mathcal{D}_{a}(\mathcal{E})$ are dense in $H^{2}(\mathcal{E})$. A direct application of the Cauchy-Schwarz inequality shows that point evaluations are bounded operators from $H^{2}(\mathcal{E})$ to $\mathcal{E}$. Specifically,

$$
\|f(z)\| \leq \frac{\|f\|_{H^{2}(\mathcal{E})}}{\left(1-|z|^{2}\right)^{1 / 2}}, \quad z \in \mathbb{D}, f \in H^{2}(\mathcal{E}) .
$$

If $f \in H^{2}(\mathcal{E})$, then the radial boundary value $f(\zeta):=\lim _{r \rightarrow 1^{-}} f(r \zeta)$ exists for $\lambda$-a.e. $\zeta \in \mathbb{T}$. The radial boundary value function satisfies

$$
\|f\|_{H^{2}(\mathcal{E})}^{2}=\frac{1}{2 \pi} \int_{\mathbb{T}}\|f(\zeta)\|^{2} \mathrm{~d} \lambda(\zeta) .
$$

In the case where $\mathcal{E}=\mathbb{C}$, these facts will be included in any reasonable introduction to Hardy spaces. For general $\mathcal{E}$, we refer to [12, Chapter III], or [16, Chapter 4].

Given an $\mathcal{L}_{+}$-valued measure $\mu$, and an analytic function $f: \mathbb{D} \rightarrow \mathcal{E}$, we define the corresponding Dirichlet integral

$$
\mathcal{D}_{\mu}(f):=\frac{1}{\pi} \int_{\mathbb{D}}\left\langle P_{\mu}(z) f^{\prime}(z), f^{\prime}(z)\right\rangle \mathrm{d} A(z) .
$$

The integrand is non-negative, so the integral is well-defined. For $z=\rho \zeta$, $\zeta \in \mathbb{T}$, the power series expansions of $P_{\mu}$ and $f$ yield

$$
\left\langle P_{\mu}(z) f^{\prime}(z), f^{\prime}(z)\right\rangle=\sum_{n=-\infty}^{\infty} \sum_{k, l=0}^{\infty} k l\langle\hat{\mu}(n) \hat{f}(k), \hat{f}(l)\rangle \rho^{|n|+k+l-2} \zeta^{n+k-l} .
$$

Integrating this identity with respect to $\rho \mathrm{d} \rho \mathrm{d} \lambda(\zeta)$, over $0<\rho<r<1$, and $\zeta \in \mathbb{T}$, gives us the formula

$$
\begin{aligned}
& \frac{1}{\pi} \int_{r \mathbb{D}}\left\langle P_{\mu}(z) f^{\prime}(z), f^{\prime}(z)\right\rangle \mathrm{d} A(z) \\
& \quad=\sum_{k, l=0}^{\infty} \min (k, l) r^{2 \max (k, l)}\langle\hat{\mu}(l-k) \hat{f}(k), \hat{f}(l)\rangle .
\end{aligned}
$$

By monotone convergence,

$$
\mathcal{D}_{\mu}(f)=\lim _{r \rightarrow 1^{-}} \sum_{k, l=0}^{\infty} \min (k, l) r^{2 \max (k, l)}\langle\hat{\mu}(l-k) \hat{f}(k), \hat{f}(l)\rangle .
$$

If the resulting series is absolutely convergent (say if $f \in \mathcal{D}_{a}(\mathcal{E})$ ), then we may of course replace the $\lim _{r \rightarrow 1^{-}}$with an evaluation at $r=1$.

We define the harmonically weighted Dirichlet space $\mathcal{D}_{\mu}^{2}(\mathcal{E})$ as the space of functions $f \in H^{2}(\mathcal{E})$ for which $\mathcal{D}_{\mu}(f)<\infty$. In particular, $\mathcal{D}_{a}(\mathcal{E}) \subseteq \mathcal{D}_{\mu}^{2}(\mathcal{E})$ by $(2.15)$. We equip $\mathcal{D}_{\mu}^{2}(\mathcal{E})$ with the norm $\|\cdot\|_{\mathcal{D}_{\mu}^{2}(\mathcal{E})}$ given by

$$
\|f\|_{\mathcal{D}_{\mu}^{2}(\mathcal{E})}^{2}:=\|f\|_{H^{2}(\mathcal{E})}^{2}+\mathcal{D}_{\mu}(f) .
$$

If $f, g \in \mathcal{D}_{\mu}^{2}(\mathcal{E})$, then we define the sesquilinear Dirichlet integral

$$
\mathcal{D}_{\mu}(f, g):=\frac{1}{\pi} \int_{\mathbb{D}}\left\langle P_{\mu}(z) f^{\prime}(z), g^{\prime}(z)\right\rangle \mathrm{d} A(z),
$$

which is finite by the Cauchy-Schwarz inequality. 
Proposition 2.6. ([14, Corollary 3.1]) Let $\mu$ be an $\mathcal{L}_{+}$-valued measure. Then $\mathcal{P}_{a}(\mathcal{E})$ is dense in the corresponding Dirichlet space $\mathcal{D}_{\mu}^{2}(\mathcal{E})$.

Proposition 2.7. Let $\mu$ be an $\mathcal{L}_{+}$-valued measure, and $f \in \mathcal{D}_{a}(\mathcal{E})$. Then

$$
\left\langle\beta_{1}\left(M_{z}\right) f, f\right\rangle_{\mathcal{D}_{\mu}^{2}(\mathcal{E})}=\frac{1}{2 \pi} \int_{\mathbb{T}}\langle\mathrm{d} \mu f, f\rangle .
$$

Proof. By (2.14), $M_{z}: H^{2}(\mathcal{E}) \rightarrow H^{2}(\mathcal{E})$ is an isometry. Hence,

$$
\left\langle\beta_{1}\left(M_{z}\right) f, f\right\rangle_{\mathcal{D}_{\mu}^{2}(\mathcal{E})}=\mathcal{D}_{\mu}^{2}\left(M_{z} f\right)-\mathcal{D}_{\mu}^{2}(f) .
$$

By (2.15),

$$
\begin{aligned}
\mathcal{D}_{\mu}^{2}\left(M_{z} f\right)-\mathcal{D}_{\mu}^{2}(f)= & \sum_{k, l=0}^{\infty} \min (k, l)\langle\hat{\mu}(l-k) \hat{f}(k-1), \hat{f}(l-1)\rangle \\
& -\sum_{k, l=0}^{\infty} \min (k, l)\langle\hat{\mu}(l-k) \hat{f}(k), \hat{f}(l)\rangle \\
= & \sum_{k, l=0}^{\infty}\langle\hat{\mu}(l-k) \hat{f}(k), \hat{f}(l)\rangle .
\end{aligned}
$$

The above right-hand side equals $\frac{1}{2 \pi} \int_{\mathbb{T}}\langle\mathrm{d} \mu f, f\rangle$ by definition.

Given an analytic function $f: \mathbb{D} \rightarrow \mathbb{C}$, and $\zeta \in \mathbb{T}$, we define the corresponding local Dirichlet integral

$$
\mathcal{D}_{\zeta}(f):=\frac{1}{\pi} \int_{\mathbb{D}}\left|f^{\prime}(z)\right|^{2} \frac{1-|z|^{2}}{|\zeta-z|^{2}} \mathrm{~d} A(z) .
$$

This is a convenient shorthand for the Dirichlet integral $\mathcal{D}_{\delta_{\zeta}}(f)$, where $\delta_{\zeta}$ denotes a (scalar) unital point mass at $\zeta$. If $\mu$ is a positive scalar-valued measure, then it is immediate from Fubini's theorem that

$$
\mathcal{D}_{\mu}(f)=\frac{1}{2 \pi} \int_{\mathbb{T}} \mathcal{D}_{\zeta}(f) \mathrm{d} \mu(\zeta) .
$$

In particular, if $\mathcal{D}_{\mu}(f)<\infty$, then $\mathcal{D}_{\zeta}(f)<\infty$ for $\mu$-a.e. $\zeta \in \mathbb{T}$.

A useful tool for calculating local Dirichlet integrals is the so-called local Douglas formula. The proof, and a slightly more general version of the statement, can be found in [7, Chapter 7.2]:

Theorem 2.8. Let $f \in H^{2}$, and $\zeta \in \mathbb{T}$. If $\mathcal{D}_{\zeta}(f)<\infty$, then the radial limit $f(\zeta)$ exists. Moreover, if we let $F(z)=\frac{f(z)-f(\zeta)}{z-\zeta}$, then $\mathcal{D}_{\zeta}(f)=\|F\|_{H^{2}}^{2}$.

Recall that a function $\theta \in H^{2}$ is called inner if $|\theta(\zeta)|=1$ for $\lambda$-a.e. $\zeta \in \mathbb{T}$. If $\theta$ is inner and $f \in \mathcal{D}_{a}$, then

$$
\mathcal{D}_{\zeta}(\theta f)=\mathcal{D}_{\zeta}(f)+|f(\zeta)|^{2} \mathcal{D}_{\zeta}(\theta)
$$

e.g. [7, Theorem 7.6.1]. For inner functions, the local Dirichlet integral $\mathcal{D}_{\zeta}(\theta)$ may be computed as

$$
\mathcal{D}_{\zeta}(\theta)=\lim _{r \rightarrow 1^{-}} \frac{1-|\theta(r \zeta)|^{2}}{1-r^{2}}
$$


e.g. [7, Theorem 7.6.5]. This formula is valid whether or not $\mathcal{D}_{\zeta}(\theta)<\infty$. If $\theta$ is analytic in a neighbourhood of $\zeta$, then $(2.19)$ yields $\mathcal{D}_{\zeta}(\theta)=\left|\theta^{\prime}(\zeta)\right|$.

In the general case of $\mathcal{L}_{+}$-valued measures, the concept of a local Dirichlet integral does not appear to be well studied. We derive the following substitute for (2.17).

Lemma 2.9. Let $\mu$ be an $\mathcal{L}_{+}$-valued measure, and $x, y \in \mathcal{E}$. If $f \in \mathcal{D}_{\mu_{x, x}}^{2}$ and $g \in \mathcal{D}_{\mu_{y, y}}^{2}$, then the integral

$$
\mathcal{D}_{\mu_{x, y}}(f, g)=\frac{1}{2 \pi^{2}} \int_{\mathbb{D}} \int_{\mathbb{T}} f^{\prime}(z) \overline{g^{\prime}(z)} \frac{1-|z|^{2}}{|\zeta-z|^{2}} \mathrm{~d} \mu_{x, y}(\zeta) \mathrm{d} A(z)
$$

is absolutely convergent, with $\left|\mathcal{D}_{\mu_{x, y}}(f, g)\right| \leq\|f\|_{\mathcal{D}_{\mu_{x, x}}^{2}}\|g\|_{\mathcal{D}_{\mu_{y, y}}^{2}}$. In particular,

$$
\mathcal{D}_{\mu_{x, y}}(f, g)=\frac{1}{2 \pi} \int_{\mathbb{T}} \mathcal{D}_{\zeta}(f, g) \mathrm{d} \mu_{x, y}(\zeta) .
$$

Proof. We need to show that

$$
\int_{\mathbb{D}} \int_{\mathbb{T}}\left|f^{\prime}(z) \overline{g^{\prime}(z)}\right| \frac{1-|z|^{2}}{|\zeta-z|^{2}} \mathrm{~d}\left|\mu_{x, y}\right|(\zeta) \mathrm{d} A(z)<\infty .
$$

The inner integral equals $\left|f^{\prime}(z) \overline{g^{\prime}(z)}\right| P_{\left|\mu_{x, y}\right|}(z)$. By an application of Lemma 2.4, we obtain

$$
P_{\left|\mu_{x, y}\right|}(z) \leq P_{\mu_{x, x}}(z)^{1 / 2} P_{\mu_{y, y}}(z)^{1 / 2} .
$$

The remainder of the statement follows from the Cauchy-Schwarz inequality, and Fubini's theorem.

\subsection{Analytic Operators}

An operator $T \in \mathcal{L}$ is called analytic if $\cap_{n \geq 0} T^{n} \mathcal{H}=\{0\}$. It is clear that an analytic operator cannot have any non-zero eigenvalues. In particular, an analytic $m$-isometry does not have eigenvalues, since $\sigma_{a p}(T) \subseteq \mathbb{T}$.

A good reason to study analytic 2-isometries is the existence of a socalled Wold decomposition: For $T \in \mathcal{L}$, we let $\mathcal{E}=\mathcal{H} \ominus T \mathcal{H}$. The dimension of $\mathcal{E}$ is called the multiplicity of $T$. Furthermore, define the spaces $\mathcal{H}_{u}=\cap_{n \geq 0} T^{n} \mathcal{H}$, and $\mathcal{H}_{a}=\bigvee_{n \geq 0} T^{n} \mathcal{E}$. The following is a special case of [19, Theorem 3.6]:

Theorem 2.10. If $T \in \mathcal{L}$ is a 2-isometry, then $\mathcal{H}=\mathcal{H}_{u} \oplus \mathcal{H}_{a}$. Moreover, the spaces $\mathcal{H}_{u}$ and $\mathcal{H}_{a}$ are invariant under $T, T_{u}:=\left.T\right|_{\mathcal{H}_{u}}$ is unitary, and $T_{a}:=\left.T\right|_{\mathcal{H}_{a}}$ is analytic.

The class of analytic 2-isometries with multiplicity 1 can be completely described in terms of $M_{z}$, multiplication by the function $z \mapsto z$, acting on harmonically weighted Dirichlet spaces [15]. This result was later generalized to arbitrary multiplicity by Olofsson [14]:

Theorem 2.11. Let $\mu$ be an $\mathcal{L}_{+}(\mathcal{E})$-valued measure. Then $M_{z}$ acts as an analytic 2 -isometry on the space $\mathcal{D}_{\mu}^{2}(\mathcal{E})$.

Conversely, suppose that $T \in \mathcal{L}(\mathcal{H})$ is an analytic 2-isometry, and let $\mathcal{E}=\mathcal{H} \ominus T \mathcal{H}$. Then there exists an $\mathcal{L}_{+}(\mathcal{E})$-valued measure $\mu$, and a unitary map $V: \mathcal{H} \rightarrow \mathcal{D}_{\mu}^{2}(\mathcal{E})$, such that $T=V^{*} M_{z} V$. 
The above correspondence is essentially one-to-one; the operators

$$
M_{z}: \mathcal{D}_{\mu_{j}}^{2}\left(\mathcal{E}_{j}\right) \rightarrow \mathcal{D}_{\mu_{j}}^{2}\left(\mathcal{E}_{j}\right), \quad j \in\{1,2\},
$$

are unitarily equivalent if and only if there exists a unitary map $U: \mathcal{E}_{1} \rightarrow \mathcal{E}_{2}$ such that $\mu_{1}(E)=U^{*} \mu_{2}(E) U$ whenever $E \in \mathfrak{S}$.

\section{3. $m$-Isometric Semigroups}

By an m-isometric semigroup we mean a $C_{0}$-semigroup $\left(T_{t}\right)_{t \geq 0}$ such that each $T_{t}$ is an $m$-isometry. In the transition from individual operators to $C_{0^{-}}$ semigroups, the following two lemmas are useful:

Lemma 3.1. Let $\left(T_{t}\right)_{t \geq 0}$ be a $C_{0}$-semigroup with generator $A$, and $m \in \mathbb{Z}_{\geq 0}$. If $y_{1}, y_{2} \in D\left(A^{m}\right)$, and $f_{y_{1}, y_{2}}:[0, \infty) \ni t \mapsto\left\langle T_{t} y_{1}, T_{t} y_{2}\right\rangle$, then $f_{y_{1}, y_{2}}$ is $m$ times differentiable, and its $m$ th derivative $f_{y_{1}, y_{2}}^{(m)}$ is given by

$$
f_{y_{1}, y_{2}}^{(m)}(t)=\alpha_{m}^{A}\left(T_{t} y_{1}, T_{t} y_{2}\right), \quad t \geq 0,
$$

where $\alpha_{m}^{A}$ is defined by (2.5). In particular, $f_{y_{1}, y_{2}}^{(m)}$ is continuous.

Proof. This is trivial for $m=0$. For $m=1$, if $y_{1}, y_{2} \in D(A), t \geq 0$, then

$$
f_{y_{1}, y_{2}}^{\prime}(t)=f_{A y_{1}, y_{2}}(t)+f_{y_{1}, A y_{2}}(t)
$$

by the product rule. By induction one obtains

$$
f_{y_{1}, y_{2}}^{(m)}(t)=\sum_{k=0}^{m}\left(\begin{array}{c}
m \\
k
\end{array}\right) f_{A^{k} y_{1}, A^{m-k} y_{2}}(t)
$$

provided that $y_{1}, y_{2} \in D\left(A^{m}\right)$. This is equivalent to (3.1).

Lemma 3.2. Let $f:[0, \infty) \rightarrow \mathbb{C}$ be a function whose mth derivative $f^{(m)}$ is continuous. For $h>0$, it then holds that

$$
\sum_{k=0}^{m}(-1)^{m-k}\left(\begin{array}{c}
m \\
k
\end{array}\right) f(k h)=\int_{s_{1}=0}^{h} \ldots \int_{s_{m}=0}^{h} f^{(m)}\left(s_{1}+\ldots+s_{m}\right) \mathrm{d} s_{1} \ldots \mathrm{d} s_{m} .
$$

Proof. Use the fundamental theorem of calculus, (2.1), and induction over $m$.

As a first application of these identities, we prove a semigroup analogue of (2.4). This is more precise than condition (ii) of [5, Theorem 2.1]:

Theorem 3.3. Let $\left(T_{t}\right)_{t \geq 0}$ be an m-isometric semigroup with generator $A$. Then the quadratic forms $\left(\alpha_{j}^{A}\right)_{j=0}^{m-1}$, densely defined by (2.5), are bounded. Moreover,

$$
\left\|T_{t} x\right\|^{2}=\sum_{j=0}^{m-1} \frac{t^{j}}{j !} \alpha_{j}^{A}(x), \quad x \in \mathcal{H}, t \geq 0 .
$$


Proof. For $y \in D\left(A^{j}\right)$, let $f(t)=\left\|T_{t} y\right\|^{2}$. By Lemma 3.1, $f^{(j)}(t)=\alpha_{j}^{A}\left(T_{t} y\right)$. Moreover, this is a continuous function, and by Lemma 3.2,

$$
\left\langle\beta_{j}\left(T_{t}\right) y, y\right\rangle=\int_{s_{1}=0}^{t} \ldots \int_{s_{j}=0}^{t} \alpha_{j}^{A}\left(T_{s_{1}+\ldots+s_{j}} y\right) \mathrm{d} s_{1} \ldots \mathrm{d} s_{j} .
$$

This identity implies

$$
\lim _{t \rightarrow 0^{+}} \frac{1}{t^{j}}\left\langle\beta_{j}\left(T_{t}\right) y, y\right\rangle=\alpha_{j}^{A}(y) .
$$

By (2.4), it holds for any $k \in \mathbb{Z}_{\geq 0}$ that

$$
\left\|T_{t / k}^{k} y\right\|^{2}=\sum_{j=0}^{m-1}\left(\begin{array}{c}
k \\
j
\end{array}\right)\left\langle\beta_{j}\left(T_{t / k}\right) y, y\right\rangle=\sum_{j=0}^{m-1}\left(\frac{t}{k}\right)^{j}\left(\begin{array}{c}
k \\
j
\end{array}\right) \frac{\left\langle\beta_{j}\left(T_{t / k}\right) y, y\right\rangle}{(t / k)^{j}} .
$$

If $y \in D\left(A^{m-1}\right)$, then we may let $k \rightarrow \infty$, in order to obtain

$$
\left\|T_{t} y\right\|^{2}=\sum_{j=0}^{m-1} \frac{t^{j}}{j !} \alpha_{j}^{A}(y) .
$$

We want to extend the above identity to $y \in \mathcal{H}$. For this it is sufficient to prove that each $\alpha_{j}^{A}$ is bounded. Given $m$ distinct times $\left(t_{k}\right)_{k=0}^{m-1}$, the numbers $\left(\left\|T_{t_{k}} y\right\|^{2}\right)_{k=0}^{m-1}$ uniquely determine $\alpha_{j}^{A}(y)$. By linear algebra, it even holds that $\alpha_{j}^{A}(y)=\sum_{k=0}^{m-1} a_{j k}\left\|T_{t_{k}} y\right\|^{2}$, where the numbers $\left(a_{j k}\right)$ do not depend on $y$. It follows that each $\alpha_{j}^{A}$ is a bounded quadratic form.

Together with (2.9), Theorem 3.3 implies the well-known result that if $A$ is the generator of an $m$-isometric semigroup, then $\sigma(A) \subseteq\{z \in \mathbb{C} ; \operatorname{Re} z \leq 0\}$. A novel result is the following:

Corollary 3.4. Let $\left(T_{t}\right)_{t>0}$ be an $m$-isometric semigroup with generator $A$. Then, there exists $a \in \mathbb{R}, b \in(a, \infty)$, such that

$$
W(A) \subseteq\{z \in \mathbb{C} ; a \leq \operatorname{Re} z \leq b\} .
$$

Proof. By Theorem 3.3, $\alpha_{1}^{A}(y)=2 \operatorname{Re}\langle A y, y\rangle$ defines a bounded quadratic form on $\mathcal{H}$.

Corollary 3.5. Let $\left(T_{t}\right)_{t \geq 0}$ be an m-isometric semigroup. Then there exists $w \geq 0$ such that

$$
\left\|T_{t}\right\| \leq e^{w t}, \quad t \geq 0
$$

i.e. $\left(T_{t}\right)_{t \geq 0}$ is quasicontractive with parameter $w$.

Proof. Since $\alpha_{0}^{A}=I$, Theorem 3.3 yields that

$$
\left\|T_{t}\right\|^{2} \leq 1+t p(t)
$$

for some polynomial $p$. It is clear that the right-hand side is dominated by $e^{w t}$ for some $w \geq 0$. 


\subsection{Proof of Theorem 1.1}

Suppose that $A$ is the generator of an $m$-isometric semigroup $\left(T_{t}\right)_{t \geq 0}$. Property (i) holds for any generator. By Corollary 3.5, $\left(T_{t}\right)_{t \geq 0}$ is quasicontractive for some parameter $w \geq 0$. Properties (ii) and (iii) are implied by the LumerPhillips theorem (Theorem 2.2). Since each $T_{t}$ is $m$-isometric, (3.3) implies that $\alpha_{m}^{A}$ vanishes, i.e. $A$ is $m$-skew-symmetric. This proves that the conditions (i)-(iv) are necessary.

Suppose on the other hand that $A$ satisfies (i)-(iv). The first three conditions, together with the Lumer-Phillips theorem, imply that $A$ generates a $C_{0}$-semigroup $\left(T_{t}\right)_{t>0}$, quasicontractive with parameter $w \geq 0$. The assumption that $A$ is $m$-skew-symmetric implies that each $T_{t}$ is $m$-isometric, by $(3.2)$.

Finally, if (i)-(iv) are fulfilled, so that $A$ generates an $m$-isometric $C_{0^{-}}$ semigroup $(T(t))_{t \geq 0}$, Theorem 3.3 together with (2.9) implies that $\lambda-A$ : $D(A) \rightarrow \mathcal{H}$ is invertible whenever $\lambda>0$.

\subsection{Proof of Theorem 1.2}

Let $\left(T_{t}\right)_{t \geq 0}$ be an $m$-isometric semigroup with generator $A$. By Theorem 1.1, $A$ is $m$-skew-symmetric, and $(\lambda-A)^{-1} \in \mathcal{L}$ for any $\lambda>0$. In particular, $1 \in \rho(A)$. For $T=(A+I)(A-I)^{-1}$, Lemma 2.1 implies that $T$ is $m$ isometric, and that each $y \in D(A)$ can be written as $\frac{1}{2}(T-I) x$ for precisely one $x \in \mathcal{H}$. Under this correspondence $A y=\frac{1}{2}(T+I) x$. It follows that (1.1) holds if and only if

$$
\|T x\|^{2}-\|x\|^{2}=\operatorname{Re}\langle(T+I) x,(T-I) x\rangle \leq w\|(T-I) x\|^{2}, \quad x \in \mathcal{H},
$$

i.e. (1.2) holds.

\section{2-Isometric Cogenerators}

The purpose of this section is to prove Theorem 1.3, which essentially amounts to proving Theorem 1.4.

We recall the hypothesis of Theorem 1.3: $T \in \mathcal{L}$ is a 2-isometry, $T-I$ is injective, and there exists $w \geq 0$ such that

$$
\left\langle\beta_{1}(T) x, x\right\rangle=\|T x\|^{2}-\|x\|^{2} \leq w\|(T-I) x\|^{2}, \quad x \in \mathcal{H} .
$$

The assertion that we wish to prove is that $T$ is the cogenerator of a $C_{0^{-}}$ semigroup $\left(T_{t}\right)_{t \geq 0}$, and that this is quasicontractive with parameter $w$.

The first step is a reduction to the case of analytic operators. By the Wold-decomposition (Theorem 2.10), $T=T_{u} \oplus T_{a}$, where $T_{u}$ is unitary and $T_{a}$ is analytic. Since $1 \notin \sigma_{p}(T)$, we know that $1 \notin \sigma_{p}\left(T_{u}\right)$. By [20, Chapter III, Section 8], $T_{u}$ is the cogenerator of a $C_{0}$-semigroup of unitary operators on $\mathcal{H}_{u}$. This is clearly quasicontractive for any $w \geq 0$. It therefore suffices to show that $T_{a}$ is the cogenerator of a quasicontractive $C_{0}$-semigroup on $\mathcal{H}_{a}$.

By orthogonality and invariance of the subspaces $\mathcal{H}_{u}, \mathcal{H}_{a}$, and the fact that $T_{u}$ is unitary, Theorem 2.10 further implies that (4.1) is equivalent to $\left\|T_{a} x_{a}\right\|^{2}-\left\|x_{a}\right\|^{2} \leq w\left\|\left(T_{u}-I_{u}\right) x_{u}\right\|^{2}+w\left\|\left(T_{a}-I_{a}\right) x_{a}\right\|^{2}, \quad\left(x_{u}, x_{a}\right) \in \mathcal{H}_{u} \oplus \mathcal{H}_{a}$. 
Consequently, $T$ satisfies (4.1) if and only if $T_{a}$ does. Together with the previous paragraph, this reduces the proof of Theorem 1.3 to the case where $T$ is an analytic operator.

With the additional hypothesis that $T$ is analytic, let $\mathcal{E}=\mathcal{H} \ominus T \mathcal{H}$. By Theorem 2.11, $T=V^{*} M_{z} V$, where $V: \mathcal{H} \rightarrow \mathcal{D}_{\mu}^{2}(\mathcal{E})$ is a unitary map, and $\mu$ is some $\mathcal{L}_{+}$-valued measure. It then holds that $\beta_{1}(T)=V^{*} \beta_{1}\left(M_{z}\right) V$, and (4.1) becomes

$$
\left\langle\beta_{1}\left(M_{z}\right) f, f\right\rangle_{\mathcal{D}_{\mu}^{2}(\mathcal{E})} \leq w\left\|\left(I-M_{z}\right) f\right\|_{\mathcal{D}_{\mu}^{2}(\mathcal{E})}^{2}, \quad f \in \mathcal{D}_{\mu}^{2}(\mathcal{E}) .
$$

By Proposition 2.6, it is sufficient to verify this for $f \in \mathcal{P}_{a}(\mathcal{E})$, and by Proposition 2.7, $T$ satisfies (4.1) if and only if

$$
\frac{1}{2 \pi} \int_{\mathbb{T}}\langle\mathrm{d} \mu f, f\rangle \leq w\left\|\left(I-M_{z}\right) f\right\|_{\mathcal{D}_{\mu}^{2}(\mathcal{E})}^{2}, \quad f \in \mathcal{P}_{a}(\mathcal{E}) .
$$

If we for a moment assume the validity of Theorem 1.4, then the above condition implies that $\left(M_{\phi_{t}}\right)_{t \geq 0}$ is a $C_{0}$-semigroup on $\mathcal{D}_{\mu}^{2}(\mathcal{E})$, and that this is quasicontractive with parameter $w$. Let $\left(T_{t}\right)_{t \geq 0}=\left(V^{*} M_{\phi_{t}} V\right)_{t \geq 0}$. This defines a $C_{0}$-semigroup on $\mathcal{H}$, it's quasicontractive with parameter $w$, and its cogenerator is given by $V^{*} M_{z} V=T$.

\subsection{Proof of Theorem 1.4}

Let us recall the statement of Theorem 1.4: If $\mu$ is an $\mathcal{L}_{+}$-valued measure on $\mathbb{T}$, then the following are equivalent:

(i) For every $t \geq 0, M_{\phi_{t}} \in \mathcal{L}\left(\mathcal{D}_{\mu}^{2}(\mathcal{E})\right)$, and the family $\left(M_{\phi_{t}}\right)_{t \geq 0}$ is a $C_{0^{-}}$ semigroup.

(ii) There exists $w_{1} \geq 0$ such that

$$
\frac{1}{2 \pi} \int_{\mathbb{T}}\langle\mathrm{d} \mu f, f\rangle \leq w_{1}\left\|\left(I-M_{z}\right) f\right\|_{\mathcal{D}_{\mu}^{2}(\mathcal{E})}^{2}, \quad f \in \mathcal{P}_{a}(\mathcal{E}) .
$$

(iii) The set function $\tilde{\mu}: E \mapsto \frac{1}{2 \pi} \int_{E} \frac{\mathrm{d} \mu(\zeta)}{|1-\zeta|^{2}}$ is an $\mathcal{L}_{+}$-valued measure, and there exists $w_{2} \geq 0$ such that

$$
\frac{1}{2 \pi} \int_{\mathbb{T}}\langle\mathrm{d} \tilde{\mu} f, f\rangle \leq w_{2}\|f\|_{\mathcal{D}_{\mu}^{2}(\mathcal{E})}^{2}, \quad f \in \mathcal{P}_{a}(\mathcal{E}) .
$$

If one (hence all) of the above conditions is satisfied, then the $C_{0}$-semigroup $\left(M_{\phi_{t}}\right)_{t \geq 0}$ is 2-isometric, has cogenerator $M_{z}$, and is quasicontractive for some parameter $w \geq 0$. Moreover, the optimal (smallest) values of $w, w_{1}$, and $w_{2}$ coincide.

In the case $\mathcal{E}=\mathbb{C}$, there is a quite direct proof that $(\mathrm{i}) \Leftrightarrow(\mathrm{iii})$. In the general case, we essentially use the same ideas, although they are somewhat obscured by technicalities. For this reason, we begin with a preliminary discussion of the case $\mathcal{E}=\mathbb{C}$. In general, the qualitative assertions that $\left(M_{\phi_{t}}\right)_{t \geq 0}$ is 2 -isometric and quasicontractive are fairly immediate from (i). The argument also shows that (i) $\Rightarrow$ (ii). The implications (ii) $\Rightarrow$ (iii) $\Rightarrow$ (i) require a bit more work. It will be evident that the optimal values of $w, w_{1}$, and $w_{2}$ coincide. 
$\mathcal{E}=\mathbb{C}:$ Note that $\phi_{t}: z \mapsto \exp (t(z+1) /(z-1))$ is inner, i.e. $\phi_{t} \in H^{2}$, and $\left|\phi_{t}(\zeta)\right|=1$ for $\lambda$-a.e. $\zeta \in \mathbb{T}$. By $(2.19)$,

$$
\mathcal{D}_{\zeta}\left(\phi_{t}\right)=\frac{2 t}{|1-\zeta|^{2}},
$$

with the interpretation $\frac{1}{|1-1|^{2}}=\infty$. If we assume (i), then $\mathcal{D}_{\mu}\left(\phi_{t}\right)<\infty$, so $\frac{2 t}{|1-\zeta|^{2}}$ is finite for $\mu$-a.e. $\zeta \in \mathbb{T}$. In particular, $\mu(\{1\})=0$. On the other hand, if we assume (iii), then again $\mu(\{1\})=0$. We may therefore add this condition to our hypothesis.

By (2.18)

$$
\mathcal{D}_{\zeta}\left(\phi_{t} f\right)=\mathcal{D}_{\zeta}(f)+|f(\zeta)|^{2} \frac{2 t}{|1-\zeta|^{2}},
$$

and this holds for $\mu$-a.e. $\zeta \in \mathbb{T}$. Using Fubini's theorem (2.17), the above formula implies that

$$
\mathcal{D}_{\mu}\left(\phi_{t} f\right)=\mathcal{D}_{\mu}(f)+\frac{t}{\pi} \int_{\mathbb{T}} \frac{|f(\zeta)|^{2}}{|1-\zeta|^{2}} \mathrm{~d} \mu(\zeta) .
$$

Adding $\left\|\phi_{t} f\right\|_{H^{2}}^{2}=\|f\|_{H^{2}}^{2}$ to the above, we obtain

$$
\left\|\phi_{t} f\right\|_{\mathcal{D}_{\mu}^{2}}^{2}=\|f\|_{\mathcal{D}_{\mu}^{2}}^{2}+\frac{t}{\pi} \int_{\mathbb{T}} \frac{|f(\zeta)|^{2}}{|1-\zeta|^{2}} \mathrm{~d} \mu(\zeta) .
$$

From this, we conclude that $M_{\phi_{t}}: \mathcal{D}_{\mu}^{2} \rightarrow \mathcal{D}_{\mu}^{2}$ is bounded for all $t \geq 0$ if and only if

$$
\frac{1}{\pi} \int_{\mathbb{T}} \frac{|f(\zeta)|^{2}}{|1-\zeta|^{2}} \mathrm{~d} \mu(\zeta) \lesssim\|f\|_{\mathcal{D}_{\mu}^{2}}^{2}, \quad f \in \mathcal{P}_{a} .
$$

For the verification that, under such circumstances, $\left(M_{\phi_{t}}\right)_{t \geq 0}$ is indeed a $C_{0}$-semigroup, we refer to the general case below.

Remark 4.1. The above argument shows that for $\mathcal{E}=\mathbb{C}$, condition (i) may be weakened to:

$\left(i^{\prime}\right)$ There exists $t>0$ such that $M_{\phi_{t}} \in \mathcal{L}\left(\mathcal{D}_{\mu}^{2}\right)$.

The author has not found a proof that the same phenomenon occurs in the general case.

(i) $\Rightarrow$ (ii) : Assume that $\left(M_{\phi_{t}}\right)_{t \geq 0} \subset \mathcal{L}\left(\mathcal{D}_{\mu}^{2}(\mathcal{E})\right)$ is a $C_{0}$-semigroup. A simple calculation shows that $M_{z}$ is the cogenerator of $\left(M_{\phi_{t}}\right)_{t \geq 0} . M_{z}$ is 2-isometric, and by Lemma 2.1, the corresponding generator is 2skew-symmetric. By Theorem 1.1, $\left(M_{\phi_{t}}\right)_{t \geq 0}$ is 2-isometric. By Corollary 3.5, $\left(M_{\phi_{t}}\right)_{t \geq 0}$ is quasicontractive for some $w \geq 0$. For any such $w$, Theorem 1.2 implies that

$$
\left\langle\beta_{1}\left(M_{z}\right) f, f\right\rangle_{\mathcal{D}_{\mu}^{2}(\mathcal{E})} \leq w\left\|\left(M_{z}-I\right) f\right\|_{\mathcal{D}_{\mu}^{2}(\mathcal{E})}^{2}, \quad f \in \mathcal{D}_{\mu}^{2}(\mathcal{E}) .
$$

In particular, the above inequality is satisfied for $f \in \mathcal{P}_{a}(\mathcal{E})$. By Proposition 2.7, condition (ii) holds with $w_{1}=w$. 
(ii) $\Rightarrow$ (iii) : Consider a fixed $w_{1} \geq 0$, and assume that

$$
\frac{1}{2 \pi} \int_{\mathbb{T}}\langle\mathrm{d} \mu f, f\rangle \leq w_{1}\left\|\left(I-M_{z}\right) f\right\|_{\mathcal{D}_{\mu}^{2}(\mathcal{E})}^{2}, \quad f \in \mathcal{P}_{a}(\mathcal{E}) .
$$

For $f \in \mathcal{D}_{a}(\mathcal{E})$, we let $f_{N}(z)=\sum_{k=0}^{N} \hat{f}(k) z^{k}$. Clearly, $\lim _{N \rightarrow \infty}$ $\left\|f_{N}\right\|_{H^{2}(\mathcal{E})}^{2}=\|f\|_{H^{2}(\mathcal{E})}^{2}$. Applying the above inequality to $f_{N} \in \mathcal{P}_{a}(\mathcal{E})$, and letting $N \rightarrow \infty$, the formulas (2.12) and (2.15) imply

$$
\frac{1}{2 \pi} \int_{\mathbb{T}}\langle\mathrm{d} \mu f, f\rangle \leq w_{1}\left\|\left(I-M_{z}\right) f\right\|_{\mathcal{D}_{\mu}^{2}(\mathcal{E})}^{2}, \quad f \in \mathcal{D}_{a}(\mathcal{E}),
$$

We will show that (4.3) implies

$$
\frac{1}{2 \pi} \int_{\mathbb{T}}\left\langle\frac{\mathrm{d} \mu(\zeta)}{|1-\zeta|^{2}} f(\zeta), f(\zeta)\right\rangle \leq w_{1}\|f\|_{\mathcal{D}_{\mu}^{2}(\mathcal{E})}^{2}, \quad f \in \mathcal{P}_{a}(\mathcal{E}) .
$$

In particular, if condition (ii) holds for some $w_{1}$, then condition (iii) holds with $w_{2}=w_{1}$.

Let $f \in \mathcal{P}_{a}(\mathcal{E})$. In order to prove (4.4), we apply (4.3) to the function $z \mapsto \frac{f(z)}{1-r z}$, where $0<r<1$. If we let $k_{r}: z \mapsto \frac{1-z}{1-r z}$, then (4.3) implies

$$
\frac{1}{2 \pi} \int_{\mathbb{T}}\left\langle\mathrm{d} \mu(\zeta) \frac{f(\zeta)}{1-r \zeta}, \frac{f(\zeta)}{1-r \zeta}\right\rangle \leq w_{1}\left\|k_{r} f\right\|_{H^{2}(\mathcal{E})}^{2}+w_{1} \mathcal{D}_{\mu}\left(k_{r} f\right) .
$$

The proof is finished by verifying the three statements

$$
\begin{gathered}
\lim _{r \rightarrow 1^{-}}\left\|k_{r} f\right\|_{H^{2}(\mathcal{E})}^{2}=\|f\|_{H^{2}(\mathcal{E})}^{2}, \\
\lim _{r \rightarrow 1^{-}} \frac{1}{2 \pi} \int_{\mathbb{T}}\left\langle\mathrm{d} \mu(\zeta) \frac{f(\zeta)}{1-r \zeta}, \frac{f(\zeta)}{1-r \zeta}\right\rangle=\frac{1}{2 \pi} \int_{\mathbb{T}}\left\langle\frac{\mathrm{d} \mu(\zeta)}{|1-\zeta|^{2}} f(\zeta), f(\zeta)\right\rangle,
\end{gathered}
$$

and

$$
\lim _{r \rightarrow 1^{-}} \mathcal{D}_{\mu}\left(k_{r} f\right)=\mathcal{D}_{\mu}(f) .
$$

For any $z \in \overline{\mathbb{D}} \backslash\{1\}$, it holds that $\left|k_{r}(z)\right| \leq \frac{2}{1+r}$, and $\lim _{r \rightarrow 1^{-}} k_{r}(z)=1$. By (2.14), and dominated convergence, (4.5) holds for any $f \in H^{2}(\mathcal{E})$.

For the verification of (4.6), an important step is to show that $\frac{\mathrm{d} \mu(\zeta)}{|1-\zeta|^{2}}$ does indeed define a measure, so that the right-hand side is well-defined. We begin with a lemma, based on the local Douglas formula:

Lemma 4.2. Let $f \in H^{2}$, and $\zeta \in \mathbb{T}$. If $\mathcal{D}_{\zeta}(f)<\infty$, then

$$
\mathcal{D}_{\zeta}\left(k_{r} f\right) \leq \frac{8}{(1+r)^{2}} \mathcal{D}_{\zeta}(f)+2 \frac{1-r}{1+r} \frac{|f(\zeta)|^{2}}{|1-r \zeta|^{2}} .
$$

Moreover, if we let $F(z)=\frac{f(z)-f(\zeta)}{z-\zeta}$, then

$$
\mathcal{D}_{\zeta}\left(f-k_{r} f\right) \leq 2\left(\left\|F-k_{r} F\right\|_{H^{2}}^{2}+\frac{1-r}{1+r} \frac{|f(\zeta)|^{2}}{|1-r \zeta|^{2}}\right) .
$$

In particular, if $\zeta \neq 1$, then $\lim _{r \rightarrow 1^{-}} \mathcal{D}_{\zeta}\left(k_{r} f\right)=\mathcal{D}_{\zeta}(f)$. 
Proof. Theorem 2.8 already asserts that $f(\zeta)$ exists, and $\mathcal{D}_{\zeta}(f)=\|F\|_{H^{2}}^{2}$. Applying the same formula to $k_{r} f$,

$$
\begin{aligned}
\mathcal{D}_{\zeta}\left(k_{r} f\right) & =\left\|\frac{k_{r}(z) f(z)-k_{r}(\zeta) f(\zeta)}{z-\zeta}\right\|_{H^{2}}^{2} \\
& =\left\|k_{r}(z) \frac{f(z)-f(\zeta)}{z-\zeta}+\frac{k_{r}(z)-k_{r}(\zeta)}{z-\zeta} f(\zeta)\right\|_{H^{2}}^{2} \\
& =\left\|k_{r}(z) F(z)-\frac{1-r}{(1-r z)(1-r \zeta)} f(\zeta)\right\|_{H^{2}}^{2} \\
& \leq 2\left(\left\|k_{r} F\right\|_{H^{2}}^{2}+\left\|\frac{1-r}{(1-r z)(1-r \zeta)} f(\zeta)\right\|_{H^{2}}^{2}\right) \\
& \leq 2\left(\frac{4}{(1+r)^{2}}\|F\|_{H^{2}}^{2}+(1-r)^{2} \frac{|f(\zeta)|^{2}}{|1-r \zeta|^{2}}\left\|\frac{1}{1-r z}\right\|_{H^{2}}^{2}\right) \\
& =\frac{8}{(1+r)^{2}} \mathcal{D}_{\zeta}(f)+2 \frac{1-r}{1+r} \frac{|f(\zeta)|^{2}}{|1-r \zeta|^{2}} .
\end{aligned}
$$

In the last step, we have used geometric summation to compute $\left\|\frac{1}{1-r z}\right\|_{H^{2}}^{2}=$ $\frac{1}{1-r^{2}}$. This proves the first inequality of the statement. The second inequality follows from a similar calculation.

The next lemma essentially states that if (4.3) holds, then a slightly weaker version of (4.4) holds for any $f$ with values in a one-dimensional subspace.

Lemma 4.3. Let $\mu$ be an $\mathcal{L}_{+}$-valued measure that satisfies (4.3). Then

$$
\frac{1}{2 \pi} \int_{\mathbb{T}} \frac{|f(\zeta)|^{2}}{|1-\zeta|^{2}} \mathrm{~d} \mu_{x, x}(\zeta) \leq w_{1}\left(\|f\|_{H^{2}}^{2}\|x\|^{2}+2 \mathcal{D}_{\mu_{x, x}}(f)\right), \quad x \in \mathcal{E}, f \in \mathcal{P}_{a} .
$$

In particular, the set function $E \mapsto \int_{E} \frac{\mathrm{d} \mu(\zeta)}{|1-\zeta|^{2}}$ is an $\mathcal{L}_{+}$-valued measure, and $\mu(\{1\})=0$.

Proof. Applying (4.3) to the function $z \mapsto \frac{f(z)}{1-r z} x$ yields

$$
\frac{1}{2 \pi} \int_{\mathbb{T}} \frac{|f(\zeta)|^{2}}{|1-r \zeta|^{2}} \mathrm{~d} \mu_{x, x}(\zeta) \leq w_{1}\left(\left\|k_{r} f\right\|_{H^{2}}^{2}\|x\|^{2}+\mathcal{D}_{\mu_{x, x}}\left(k_{r} f\right)\right) .
$$

Using Lemma 4.2, together with Fubini's theorem (2.17),

$$
\mathcal{D}_{\mu_{x, x}}\left(k_{r} f\right) \leq \frac{8}{(1+r)^{2}} \mathcal{D}_{\mu_{x, x}}(f)+\frac{1}{\pi} \frac{1-r}{1+r} \int_{\mathbb{T}} \frac{|f(\zeta)|^{2}}{|1-r \zeta|^{2}} \mathrm{~d} \mu_{x, x}(\zeta) .
$$

Thus, subtracting $\frac{w_{1}}{\pi} \frac{1-r}{1+r} \int_{\mathbb{T}} \frac{|f(\zeta)|^{2}}{|1-r \zeta|^{2}} \mathrm{~d} \mu_{x, x}(\zeta)$ from both sides of (4.8) yields

$$
\begin{aligned}
& \left(\frac{1}{2 \pi}-\frac{w_{1}}{\pi} \frac{1-r}{1+r}\right) \int_{\mathbb{T}} \frac{|f(\zeta)|^{2}}{|1-r \zeta|^{2}} \mathrm{~d} \mu_{x, x}(\zeta) \\
& \quad \leq w_{1}\left(\left\|k_{r} f\right\|_{H^{2}}^{2}\|x\|^{2}+\frac{8}{(1+r)^{2}} \mathcal{D}_{\mu_{x, x}}(f)\right) .
\end{aligned}
$$


A formal application of Fatou's lemma yields

$$
\begin{aligned}
\frac{1}{2 \pi} \int_{\mathbb{T}} \frac{|f(\zeta)|^{2}}{|1-\zeta|^{2}} \mathrm{~d} \mu_{x, x}(\zeta) & =\frac{1}{2 \pi} \int_{\mathbb{T}} \liminf _{r \rightarrow 1^{-}} \frac{|f(\zeta)|^{2}}{|1-r \zeta|^{2}} \mathrm{~d} \mu_{x, x}(\zeta) \\
& \leq \liminf _{r \rightarrow 1^{-}} \frac{1}{2 \pi} \int_{\mathbb{T}} \frac{|f(\zeta)|^{2}}{|1-r \zeta|^{2}} \mathrm{~d} \mu_{x, x}(\zeta) \\
& =\liminf _{r \rightarrow 1^{-}}\left(\frac{1}{2 \pi}-\frac{w_{1}}{\pi} \frac{1-r}{1+r}\right) \int_{\mathbb{T}} \frac{|f(\zeta)|^{2}}{|1-r \zeta|^{2}} \mathrm{~d} \mu_{x, x}(\zeta) \\
& \leq \liminf _{r \rightarrow 1^{-}} w_{1}\left(\left\|k_{r} f\right\|_{H^{2}}^{2}\|x\|^{2}+\frac{8}{(1+r)^{2}} \mathcal{D}_{\mu_{x, x}}(f)\right) \\
& =w_{1}\left(\|f\|_{H^{2}}^{2}\|x\|^{2}+2 \mathcal{D}_{\mu_{x, x}}(f)\right) .
\end{aligned}
$$

In order to justify this, we need to argue that $\frac{|f(\zeta)|^{2}}{|1-\zeta|^{2}}=\lim _{r \rightarrow 1^{-}} \frac{|f(\zeta)|^{2}}{|1-r \zeta|^{2}}$ for $\mu_{x, x}$-a.e. $\zeta \in \mathbb{T}$. For the constant function $f \equiv 1$ we have convergence for every $\zeta$, and we obtain

$$
\frac{1}{2 \pi} \int_{\mathbb{T}} \frac{1}{|1-\zeta|^{2}} \mathrm{~d} \mu_{x, x}(\zeta) \leq w_{1}\|x\|^{2}, \quad x \in \mathcal{E} .
$$

This implies that $\frac{1}{|1-\zeta|^{2}}$ is finite for $\mu_{x, x}$-a.e. $\zeta \in \mathbb{T}$. As this holds for every $x \in \mathcal{E}, \mu(\{1\})=0$. This justifies the above argument for any $f \in \mathcal{P}_{a}(\mathcal{E})$.

As was noted in Sect. 2.3, the above inequality also implies that $E \mapsto$ $\int_{E} \frac{\mathrm{d} \mu(\zeta)}{|1-\zeta|^{2}}$ is an $\mathcal{L}_{+}$-valued measure.

By hypothesis (4.3) holds, so Lemma 4.3 allows us to assume that $\mu(\{1\})=0$.

We now prove (4.6). By Lemma 4.3, the right-hand side is well-defined. For $f \in \mathcal{P}_{a}(\mathcal{E})$, let $\left\{e_{n}\right\} \subset \mathcal{E}$ be an orthonormal basis of a finite-dimensional subspace containing the range of $f$. Then $f=\sum_{n} f_{n} e_{n}$, where each $f_{n} \in \mathcal{P}_{a}$. Defining $\mu_{m, n}=\mu_{e_{m}, e_{n}}$, a calculation shows that

$$
\int_{\mathbb{T}}\left\langle\mathrm{d} \mu(\zeta) \frac{f(\zeta)}{1-r \zeta}, \frac{f(\zeta)}{1-r \zeta}\right\rangle=\sum_{m, n} \int_{\mathbb{T}} \frac{f_{m}(\zeta) \overline{f_{n}(\zeta)}}{|1-r \zeta|^{2}} \mathrm{~d} \mu_{m, n}(\zeta) .
$$

For $\left|\mu_{m, n}\right|$-a.e. $\zeta \in \mathbb{T}$,

$$
\lim _{r \rightarrow 1^{-}} \frac{f_{m}(\zeta) \overline{f_{n}(\zeta)}}{|1-r \zeta|^{2}}=\frac{f_{m}(\zeta) \overline{f_{n}(\zeta)}}{|1-\zeta|^{2}}
$$

Note that

$$
\frac{\left|f_{m}(\zeta) \overline{f_{n}(\zeta)}\right|}{|1-r \zeta|^{2}} \leq 4 \frac{\left|f_{m}(\zeta) \overline{f_{n}(\zeta)}\right|}{|1-\zeta|^{2}}
$$

By Lemmas 2.4, and 4.3, the above right-hand side is $\left|\mu_{m, n}\right|$-integrable. Hence, by dominated convergence, 


$$
\begin{gathered}
\int_{\mathbb{T}}\left\langle\mathrm{d} \mu(\zeta) \frac{f(\zeta)}{1-r \zeta}, \frac{f(\zeta)}{1-r \zeta}\right\rangle=\sum_{m, n} \int_{\mathbb{T}} \frac{f_{m}(\zeta) \overline{f_{n}(\zeta)}}{|1-r \zeta|^{2}} \mathrm{~d} \mu_{m, n}(\zeta) \\
\rightarrow \sum_{m, n} \int_{\mathbb{T}} \frac{f_{m}(\zeta) \overline{f_{n}(\zeta)}}{|1-\zeta|^{2}} \mathrm{~d} \mu_{m, n}(\zeta)=\int_{\mathbb{T}}\left\langle\frac{\mathrm{d} \mu(\zeta)}{|1-\zeta|^{2}} f(\zeta), f(\zeta)\right\rangle .
\end{gathered}
$$

This proves that if $f \in \mathcal{P}_{a}(\mathcal{E})$, and $\mu$ satisfies (4.3), then (4.6) holds.

The proof of (4.7) is similar. Reusing the above notation,

$$
\mathcal{D}_{\mu}\left(k_{r} f\right)=\sum_{m, n} \mathcal{D}_{\mu_{m, n}}\left(k_{r} f_{m}, k_{r} f_{n}\right) .
$$

By Lemma 2.9, each one of these Dirichlet integrals can be computed as

$$
\mathcal{D}_{\mu_{m, n}}\left(k_{r} f_{m}, k_{r} f_{n}\right)=\frac{1}{2 \pi} \int_{\mathbb{T}} \mathcal{D}_{\zeta}\left(k_{r} f_{m}, k_{r} f_{n}\right) \mathrm{d} \mu_{m, n}(\zeta) .
$$

By polarization, and Lemma 4.2,

$$
\begin{aligned}
\mathcal{D}_{\zeta}\left(k_{r} f_{m}, k_{r} f_{n}\right)= & \frac{1}{4} \sum_{\sigma^{4}=1} \sigma \mathcal{D}_{\zeta}\left(k_{r}\left(f_{m}+\sigma f_{n}\right)\right) \\
& \rightarrow \frac{1}{4} \sum_{\sigma^{4}=1} \sigma \mathcal{D}_{\zeta}\left(f_{m}+\sigma f_{n}\right)=\mathcal{D}_{\zeta}\left(f_{m}, f_{n}\right),
\end{aligned}
$$

for $\left|\mu_{m, n}\right|$-a.e $\zeta \in \mathbb{T}$. Moreover,

$$
\begin{aligned}
& \left|\mathcal{D}_{\zeta}\left(k_{r} f_{m}, k_{r} f_{n}\right)\right| \leq \mathcal{D}_{\zeta}\left(k_{r} f_{m}\right)^{1 / 2} \mathcal{D}_{\zeta}\left(k_{r} f_{n}\right)^{1 / 2} \\
& \quad \leq\left(8 \mathcal{D}_{\zeta}\left(f_{m}\right)+4 \frac{\left|f_{m}(\zeta)\right|^{2}}{|1-\zeta|^{2}}\right)^{1 / 2}\left(8 \mathcal{D}_{\zeta}\left(f_{n}\right)+4 \frac{\left|f_{n}(\zeta)\right|^{2}}{|1-\zeta|^{2}}\right)^{1 / 2}
\end{aligned}
$$

By Lemmas 2.4, and 4.3, the right-hand side is $\left|\mu_{m, n}\right|$-integrable. By dominated convergence,

$$
\begin{aligned}
\mathcal{D}_{\mu}\left(k_{r} f\right)= & \sum_{m, n} \frac{1}{2 \pi} \int_{\mathbb{T}} \mathcal{D}_{\zeta}\left(k_{r} f_{m}, k_{r} f_{n}\right) \mathrm{d} \mu_{m, n}(\zeta) \\
& \rightarrow \sum_{m, n} \frac{1}{2 \pi} \int_{\mathbb{T}} \mathcal{D}_{\zeta}\left(f_{m}, f_{n}\right) \mathrm{d} \mu_{m, n}(\zeta)=\mathcal{D}_{\mu}(f) .
\end{aligned}
$$

This shows that (4.7) holds whenever $f \in \mathcal{P}_{a}(\mathcal{E})$, and $\mu$ satisfies (4.3). The proof that (ii) $\Rightarrow$ (iii) is complete.

(iii) $\Rightarrow$ (i) : We are assuming the existence of $w_{2} \geq 0$, such that

$$
\frac{1}{2 \pi} \int_{\mathbb{T}}\left\langle\frac{\mathrm{d} \mu(\zeta)}{|1-\zeta|^{2}} f(\zeta), f(\zeta)\right\rangle \leq w_{2}\|f\|_{\mathcal{D}_{\mu}^{2}(\mathcal{E})}^{2}, \quad f \in \mathcal{P}_{a}(\mathcal{E}) .
$$

Recall that $\phi_{t}: z \mapsto \exp (t(z+1) /(z-1))$. The core of our proof is the following formula:

Lemma 4.4. Let $\mu$ be an $\mathcal{L}_{+}$-valued measure that satisfies (4.9). If $f \in \mathcal{P}_{a}(\mathcal{E})$, and $t>0$, then

$$
\left\|\phi_{t} f\right\|_{\mathcal{D}_{\mu}^{2}(\mathcal{E})}^{2}=\|f\|_{\mathcal{D}_{\mu}^{2}(\mathcal{E})}^{2}+\frac{t}{\pi} \int_{\mathbb{T}}\left\langle\frac{\mathrm{d} \mu(\zeta)}{|1-\zeta|^{2}} f(\zeta), f(\zeta)\right\rangle .
$$


Proof. Reusing some previous notation, $f=\sum f_{n} e_{n}$, where each $f_{n} \in \mathcal{P}_{a}$, and $\left\{e_{n}\right\}$ is a finite orthonormal set. Moreover, $\mu_{m, n}=\mu_{e_{m}, e_{n}}$. From our discussion of the case $\mathcal{E}=\mathbb{C}$, in particular (4.2), we know that

$$
\mathcal{D}_{\mu_{n, n}}\left(\phi_{t} f_{n}\right)=\mathcal{D}_{\mu_{n, n}}\left(f_{n}\right)+\frac{t}{\pi} \int_{\mathbb{T}} \frac{\left|f_{n}(\zeta)\right|^{2}}{|1-\zeta|^{2}} \mathrm{~d} \mu_{n, n}(\zeta) .
$$

Applying (4.9) to the function $f_{n} e_{n}$, the last integral is finite, so $\phi_{t} f_{n} \in \mathcal{D}_{\mu_{n, n}}^{2}$, or equivalently, $\phi_{t} f_{n} e_{n} \in \mathcal{D}_{\mu}^{2}(\mathcal{E})$. By the Cauchy-Schwarz inequality, this implies that each integral $\mathcal{D}_{\mu}\left(\phi_{t} f_{m} e_{m}, \phi_{t} f_{n} e_{n}\right)$ is finite, as is

$$
\int_{\mathbb{T}} \frac{f_{m}(\zeta) \overline{f_{n}(\zeta)}}{|1-\zeta|^{2}} \mathrm{~d} \mu_{m, n}(\zeta)
$$

Summing over $m$ and $n$ yields

$$
\mathcal{D}_{\mu}\left(\phi_{t} f\right)=\mathcal{D}_{\mu}(f)+\frac{t}{\pi} \int_{\mathbb{T}}\left\langle\frac{\mathrm{d} \mu(\zeta)}{|1-\zeta|^{2}} f(\zeta), f(\zeta)\right\rangle .
$$

To reach the desired conclusion, we add $\left\|\phi_{t} f\right\|_{H^{2}(\mathcal{E})}^{2}=\|f\|_{H^{2}(\mathcal{E})}^{2}$ on both sides.

Let $t>0$. By Lemma 4.4, $M_{\phi_{t}}: \mathcal{P}_{a}(\mathcal{E}) \rightarrow \mathcal{D}_{\mu}^{2}(\mathcal{E})$ is $\mathcal{D}_{\mu}^{2}(\mathcal{E})$-bounded. As such, it has a unique bounded extension $\tilde{M}: \mathcal{D}_{\mu}^{2}(\mathcal{E}) \rightarrow \mathcal{D}_{\mu}^{2}(\mathcal{E})$. By (2.13), if $f_{n} \rightarrow f$ in $H^{2}(\mathcal{E})$, then $f_{n}(z) \rightarrow f(z)$ for each $z \in \mathbb{D}$. Since convergence in $\mathcal{D}_{\mu}^{2}(\mathcal{E})$ implies convergence in $H^{2}(\mathcal{E})$, we obtain that $\tilde{M}$ is indeed given by multiplication by $\phi_{t}$. Hence, $\left(M_{\phi_{t}}\right)_{t \geq 0} \subset \mathcal{L}\left(\mathcal{D}_{\mu}^{2}(\mathcal{E})\right)$. It is clear that $\left(M_{\phi_{t}}\right)_{t \geq 0}$ is a semigroup.

We need to prove that $\left(M_{\phi_{t}}\right)_{t \geq 0}$ is strongly continuous. If $f \in \mathcal{P}_{a}(\mathcal{E})$, then Lemma 4.4 implies that

$$
\left\|\phi_{t} f\right\|_{\mathcal{D}_{\mu}^{2}(\mathcal{E})}^{2}=\|f\|_{\mathcal{D}_{\mu}^{2}(\mathcal{E})}^{2}+t\left\langle\beta_{1}\left(M_{\phi_{1}}\right) f, f\right\rangle_{\mathcal{D}_{\mu}^{2}(\mathcal{E})},
$$

and by continuity, this identity extends to $f \in \mathcal{D}_{\mu}^{2}(\mathcal{E})$. We conclude that, for fixed $f \in \mathcal{D}_{\mu}^{2}(\mathcal{E})$, the family $\left(\phi_{t} f\right)_{0<t<1}$ is bounded in $\mathcal{D}_{\mu}^{2}(\mathcal{E})$. As we let $t \rightarrow 0^{+}$, a subsequence of $\left(\phi_{t} f\right)_{0<t<1}$ will converge weakly to some $g \in \mathcal{D}_{\mu}^{2}(\mathcal{E})$. If $z \in \mathbb{D}$, and $x \in \mathcal{E}$, then (2.13) implies that $f \mapsto\langle f(z), x\rangle$ is a bounded linear functional on $\mathcal{D}_{\mu}^{2}(\mathcal{E})$, so $\left\langle\phi_{t} f(z), x\right\rangle \rightarrow\langle g(z), x\rangle$ for said subsequence. But the point-wise limit of $\phi_{t} f$ is $f$, so $g=f$. As this uniquely determines the limit of any subsequence, the entire family converges weakly to $f$. Since (4.10) also implies that $\lim _{t \rightarrow 0^{+}}\left\|\phi_{t} f\right\|_{\mathcal{D}_{\mu}^{2}(\mathcal{E})}^{2}=\|f\|_{\mathcal{D}_{\mu}^{2}(\mathcal{E})}^{2}$, we conclude that $\lim _{t \rightarrow 0^{+}} \phi_{t} f=f$ with convergence in $\mathcal{D}_{\mu}^{2}(\mathcal{E})$. This establishes that $\left(M_{\phi_{t}}\right)_{t \geq 0}$ is a $C_{0}$-semigroup, i.e. (iii) $\Rightarrow(\mathrm{i})$.

It remains to prove that $\left(M_{\phi_{t}}\right)_{t \geq 0}$ is quasicontractive, with parameter $w_{2}$. It follows from (4.9) and Lemma 4.4 that

$$
\left\|M_{\phi_{t}}\right\|^{2} \leq 1+2 w_{2} t, \quad t \geq 0 .
$$

The right-hand side is the tangent at $t=0$ of the convex function $t \mapsto$ $\exp \left(2 w_{2} t\right)$. Hence, $\left(M_{\phi_{t}}\right)_{t \geq 0}$ is quasicontractive with parameter $w_{2}$. 


\section{Examples}

The following example establishes the existence of 2-isometric semigroups:

Example. Let $h: \mathbb{T} \rightarrow \mathcal{L}_{+}$be a function such that $\zeta \mapsto \frac{\|h(\zeta)\|}{|1-\zeta|^{2}}$ is bounded, and $\zeta \mapsto\langle h(\zeta) x, y\rangle$ is $\lambda$-measurable for each $x, y \in \mathcal{E}$. Defining $\mu \geq 0$ by $\langle\mu(E) x, y\rangle=\int_{E}\langle h(\zeta) x, y\rangle \mathrm{d} \lambda(\zeta)$, we have that

$$
\begin{aligned}
& \frac{1}{2 \pi} \int_{\mathbb{T}}\left\langle\frac{\mathrm{d} \mu(\zeta)}{|1-\zeta|^{2}} f(\zeta), f(\zeta)\right\rangle=\frac{1}{2 \pi} \int_{\mathbb{T}} \frac{\|h(\zeta) f(\zeta)\|^{2}}{|1-\zeta|^{2}} \mathrm{~d} \lambda(\zeta) \\
& \quad \lesssim\|f\|_{H^{2}(\mathcal{E})}^{2} \leq\|f\|_{\mathcal{D}_{\mu}^{2}(\mathcal{E})}^{2}, \quad f \in \mathcal{P}_{a}(\mathcal{E}) .
\end{aligned}
$$

Therefore, condition (iii) of Theorem 1.4 is satisfied, and $\left(M_{\phi_{t}}\right)_{t \geq 0} \subset \mathcal{L}\left(\mathcal{D}_{\mu}^{2}(\mathcal{E})\right)$ is a 2-isometric semigroup.

Not every 2-isometry is the cogenerator of a $C_{0}$-semigroup:

Example. Let $\mathcal{E}=\mathbb{C}$, and $\mu=\lambda$. Then condition (iii) of Theorem 1.4 is not satisfied, so $M_{z}: \mathcal{D}_{\lambda}^{2} \rightarrow \mathcal{D}_{\lambda}^{2}$ is not the cogenerator of a $C_{0}$-semigroup. Identifying an analytic function with its sequence of Maclaurin coefficients, $\mathcal{D}_{\lambda}^{2}$ is isometrically isomorphic to the space of sequences $\left(a_{k}\right)_{k \geq 0}$ such that

$$
\sum_{k=0}^{\infty}(1+k)\left|a_{k}\right|^{2}<\infty
$$

e.g. [7, Chapter 1]. Under this identification, $M_{z}: \mathcal{D}_{\lambda}^{2} \rightarrow \mathcal{D}_{\lambda}^{2}$ is unitarily equivalent to the right-shift

$$
\left(a_{0}, a_{1}, a_{2}, \ldots\right) \mapsto\left(0, a_{0}, a_{1}, \ldots\right) .
$$

Hence, the operator $M_{z}: \mathcal{D}_{\lambda}^{2} \rightarrow \mathcal{D}_{\lambda}^{2}$ is arguably the simplest example of a non-isometric 2-isometry.

The above example shows that $M_{z}: \mathcal{D}_{\lambda}^{2} \rightarrow \mathcal{D}_{\lambda}^{2}$ does not satisfy (1.2) for any $w \geq 0$. Hence, the corresponding condition in Theorem 1.3 is not superfluous. Similarly, condition (ii) of Theorem 1.1 is also not superfluous. However, in order to see this we need a slightly more refined example:

Recall that $M_{z}-I: \mathcal{D}_{\mu}^{2}(\mathcal{E}) \rightarrow \mathcal{D}_{\mu}^{2}(\mathcal{E})$ is injective for any operator measure $\mu \geq 0$, see Sect. 2.5. We may therefore define the operator

$$
A=\left(M_{z}+I\right)\left(M_{z}-I\right)^{-1}, \quad D(A)=\left(M_{z}-I\right) \mathcal{D}_{\mu}^{2}(\mathcal{E}) .
$$

Since $A-I=2\left(M_{z}-I\right)^{-1}$ has the bounded inverse $\frac{1}{2}\left(M_{z}-I\right)$, we conclude that $A$ is closed, and $1 \in \rho(A)$. Moreover, $M_{z}$ is 2 -isometric, so Lemma 2.1 yields that $A$ is 2 -skew-symmetric. If $\lambda>0$, then

$$
(\lambda-A)^{-1}=\frac{1}{1+\lambda}\left(M_{z}-I\right)\left(\frac{\lambda-1}{\lambda+1} M_{z}-I\right)^{-1} .
$$

The last factor exists as a bounded operator, because $\frac{\lambda-1}{\lambda+1}<1$, and $\sigma\left(M_{z}\right)=$ $\overline{\mathbb{D}}$. The operator $A$ is densely defined if and only if $M_{z}-I$ has dense range. Moreover, $A$ is the generator of a $C_{0}$-semigroup if and only if $M_{z}$ is the cogenerator. 
Example. Define $\mu$ by $\mathrm{d} \mu(\zeta)=|1-\zeta| \mathrm{d} \lambda(\zeta)$. This measure violates condition (iii) of Theorem 1.4. Hence, $M_{z}: \mathcal{D}_{\mu}^{2} \rightarrow \mathcal{D}_{\mu}^{2}$ is not the cogenerator of a $C_{0}$-semigroup.

We now prove that $M_{z}-I$ has dense range. For $z \in \overline{\mathbb{D}}$, and $r \in(0,1)$, let $k_{r}(z)=\frac{1-z}{1-r z}$. If $f \in \mathcal{D}_{\mu}^{2}$, then $k_{r} f \in\left(M_{z}-I\right) \mathcal{D}_{\mu}^{2}$. We prove that $k_{r} f \rightarrow f$ in $\mathcal{D}_{\mu}^{2}$ as $r \rightarrow 1^{-}$. It is clear that $\left\|f-k_{r} f\right\|_{H^{2}} \rightarrow 0$. Hence, we need to show that $\mathcal{D}_{\mu}\left(f-k_{r} f\right) \rightarrow 0$.

Since $f \in \mathcal{D}_{\mu}^{2}, \mathcal{D}_{\zeta}(f)<\infty$ for $\mu$-a.e. $\zeta \in \mathbb{T}$. For such $\zeta$, let $F(z)=$ $\frac{f(z)-f(\zeta)}{z-\zeta}$. From Lemma 4.2,

$$
\mathcal{D}_{\zeta}\left(f-k_{r} f\right) \leq 2\left(\left\|F-k_{r} F\right\|_{H^{2}}^{2}+\frac{1-r}{1+r} \frac{|f(\zeta)|^{2}}{|1-r \zeta|^{2}}\right) .
$$

Except possibly for $\zeta=1, \mathcal{D}_{\zeta}\left(f-k_{r} f\right) \rightarrow 0$ as $r \rightarrow 1^{-}$. Moreover, $\| F-$ $k_{r} F \|_{H^{2}}^{2}$ is bounded, while

$$
\frac{1-r}{1+r} \frac{|f(\zeta)|^{2}}{|1-r \zeta|^{2}} \leq 4 \frac{|f(\zeta)|^{2}}{|1-\zeta|}
$$

This right-hand side is $\mu$-integrable, because $\mathcal{D}_{\mu}^{2} \subset H^{2}$. By Fubini's theorem (2.17), dominated convergence implies that

$$
\mathcal{D}_{\mu}\left(f-k_{r} f\right)=\frac{1}{\pi} \int_{\mathbb{T}} \mathcal{D}_{\zeta}\left(f-k_{r} f\right) \mathrm{d} \mu(\zeta) \rightarrow 0 .
$$

Therefore, $M_{z}-I$ has dense range.

Based on our discussion, we conclude that the operator $A$, defined by

$$
A=\left(M_{z}+I\right)\left(M_{z}-I\right)^{-1}, \quad D(A)=\left(M_{z}-I\right) \mathcal{D}_{\mu}^{2},
$$

is closed, densely defined, and 2-skew-symmetric. Moreover, $\lambda-A: D(A) \rightarrow$ $\mathcal{H}$ is surjective for any $\lambda>0$. Nevertheless, $A$ fails to generate a $C_{0}$-semigroup, apparently for the sole reason that (1.1) is not satisfied for any $w \geq 0$.

In the proof of Theorem 1.4 , recall our discussion of the case $\mathcal{E}=\mathbb{C}$ on p. 18. The main part of the argument was to identify the measures $\mu$ for which the multiplication operators $\left(M_{\phi_{t}}\right)_{t \geq 0}$ are bounded on $\mathcal{D}_{\mu}^{2}$. With this in mind, we give an example of a semigroup that would appear to be 2 -isometric, apart from the fact that its elements are not bounded operators. This relates to some other recent examples of "unbounded 2-isometries", [6, Example 3.4], and [18, Example 7.1].

Example. Consider the right-shift semigroup $(S(t))_{t \geq 0}$, defined for functions $f:(0, \infty) \rightarrow \mathbb{C}$ by

$$
(S(t) f)(s)= \begin{cases}f(s-t), & s \geq t \\ 0, & t>s .\end{cases}
$$

Clearly, $S(0) f=f$, and $S\left(t_{1}\right) S\left(t_{2}\right)=S\left(t_{1}+t_{2}\right)$. Consider now the Hilbert space $L^{2}(0, \infty ; s \mathrm{~d} s)$ of functions $f:(0, \infty) \rightarrow \mathbb{C}$ such that

$$
\|f\|_{1}^{2}:=\int_{s=0}^{\infty}|f(s)|^{2} s \mathrm{~d} s<\infty .
$$


If $f$ is the indicator function of the interval $(0, h)$, then $\|f\|_{1}^{2}=\frac{h^{2}}{2}$, whereas $\|S(t) f\|_{1}^{2}=t h+\frac{h^{2}}{2}$. Hence, any non-trivial right-shift $S(t)$ fails to be bounded on $L^{2}(0, \infty ; s \mathrm{~d} s)$. On the other hand, if $f \in L^{2}(0, \infty ; s \mathrm{~d} s) \cap L_{l o c}^{2}(0, \infty)$, which is the natural domain of $S(t)$, then $\left\|S(t)^{2} f\right\|_{1}^{2}-2\|S(t) f\|_{1}^{2}+\|f\|_{1}^{2}=0$.

\section{6. $m$-Concave Semigroups}

Let $\left(T_{t}\right)_{t \geq 0}$ be a $C_{0}$-semigroup. We say that $\left(T_{t}\right)_{t \geq 0}$ is $m$-concave if each $T_{t}$ is $m$-concave, i.e. $\beta_{m}\left(T_{t}\right) \leq 0$. One can use (3.2) to obtain:

Proposition 6.1. Let $m \in \mathbb{Z}_{\geq 1}$, and $\left(T_{t}\right)_{t \geq 0}$ be a $C_{0}$-semigroup with generator A. Then $\left(T_{t}\right)_{t \geq 0}$ is $m$-concave if and only if

$$
\alpha_{m}^{A}(y) \leq 0, \quad y \in D\left(A^{m}\right),
$$

where $\alpha_{m}^{A}$ is given by $(2.5)$.

For $m$-concave operators, the following analogue of (2.4) holds:

Proposition 6.2. Let $m \in \mathbb{Z}_{\geq 1}$, and assume that $T \in \mathcal{L}$ is $m$-concave. Then

$$
\left\|T^{k} x\right\|^{2} \leq \sum_{j=0}^{m-1}\left(\begin{array}{l}
k \\
j
\end{array}\right)\left\langle\beta_{j}(T) x, x\right\rangle, \quad x \in \mathcal{H} .
$$

Proof. For $k<m$ we have equality, by (2.3). We therefore consider $k \geq m$ :

$$
T^{* k} T^{k}=\sum_{j=0}^{m-1}\left(\begin{array}{l}
k \\
j
\end{array}\right) \beta_{j}(T)+\sum_{j=m}^{k}\left(\begin{array}{l}
k \\
j
\end{array}\right) \beta_{j}(T) .
$$

It suffices to show that the second sum is $\leq 0$. From [18, Proposition 2.1], we take the formula

$$
\beta_{j+m}(T)=\sum_{i=0}^{j}(-1)^{j-i}\left(\begin{array}{l}
j \\
i
\end{array}\right) T^{* i} \beta_{m}(T) T^{i} .
$$

Shifting the index, and changing the order of summation, the previous formula yields

$$
\begin{aligned}
\sum_{j=m}^{k}\left(\begin{array}{l}
k \\
j
\end{array}\right) \beta_{j}(T) & =\sum_{j=0}^{k-m}\left(\begin{array}{c}
k \\
j+m
\end{array}\right) \beta_{j+m}(T) \\
& =\sum_{j=0}^{k-m}\left(\begin{array}{c}
k \\
j+m
\end{array}\right) \sum_{i=0}^{j}(-1)^{j-i}\left(\begin{array}{l}
j \\
i
\end{array}\right) T^{* i} \beta_{m}(T) T^{i} \\
& =\sum_{i=0}^{k-m}\left(\sum_{j=i}^{k-m}(-1)^{j-i}\left(\begin{array}{c}
k \\
j+m
\end{array}\right)\left(\begin{array}{l}
j \\
i
\end{array}\right)\right) T^{* i} \beta_{m}(T) T^{i}
\end{aligned}
$$

Since $\beta_{m}(T) \leq 0$ by assumption, we are done if $\sum_{j=i}^{N}(-1)^{j-i}\left(\begin{array}{c}N+m \\ j+m\end{array}\right)\left(\begin{array}{c}j \\ i\end{array}\right) \geq 0$ for all integers $i$ and $N$ with $N \geq i \geq 0$. This follows by Lemma 6.3 below. 
Lemma 6.3. Given integers $N \geq i \geq 0, m \geq 1$, it holds that

$$
\sum_{j=i}^{N}(-1)^{j-i}\left(\begin{array}{c}
N+m \\
j+m
\end{array}\right)\left(\begin{array}{l}
j \\
i
\end{array}\right)=\left(\begin{array}{c}
m-1+N-i \\
N-i
\end{array}\right) .
$$

Proof. Define

$$
\operatorname{LHS}_{i, N}^{(m)}=\sum_{j=i}^{N}(-1)^{j-i}\left(\begin{array}{c}
N+m \\
j+m
\end{array}\right)\left(\begin{array}{l}
j \\
i
\end{array}\right)
$$

and

$$
\operatorname{RHS}_{i, N}^{(m)}=\left(\begin{array}{c}
m-1+N-i \\
N-i
\end{array}\right)
$$

Step 1, $(i=0)$ : Using (2.1),

$$
\begin{aligned}
\operatorname{LHS}_{0, N}^{(m)} & =\sum_{j=0}^{N}(-1)^{j}\left[\left(\begin{array}{c}
N+m-1 \\
j+m-1
\end{array}\right)+\left(\begin{array}{c}
N+m-1 \\
j+m
\end{array}\right)\right] \\
& =\sum_{j=-1}^{N-1}(-1)^{j+1}\left(\begin{array}{c}
N+m-1 \\
j+m
\end{array}\right)+\sum_{j=0}^{N}(-1)^{j}\left(\begin{array}{c}
N+m-1 \\
j+m
\end{array}\right) \\
& =\left(\begin{array}{c}
N+m-1 \\
m-1
\end{array}\right)+(-1)^{N}\left(\begin{array}{c}
N+m-1 \\
N+m
\end{array}\right) \\
& =\left(\begin{array}{c}
N+m-1 \\
N
\end{array}\right)=\operatorname{RHS}_{0, N}^{(m)} .
\end{aligned}
$$

Step 2, (recursion formula) : Using (2.1) again,

$$
\begin{aligned}
\operatorname{LHS}_{i, N}^{(m)}= & \sum_{j=i}^{N}(-1)^{j-i}\left(\begin{array}{c}
N+m \\
j+m
\end{array}\right)\left[\left(\begin{array}{c}
j-1 \\
i-1
\end{array}\right)+\left(\begin{array}{c}
j-1 \\
i
\end{array}\right)\right] \\
= & \sum_{j=i-1}^{N-1}(-1)^{j+1-i}\left(\begin{array}{c}
N+m \\
j+m+1
\end{array}\right)\left(\begin{array}{c}
j \\
i-1
\end{array}\right) \\
& +\sum_{j=i}^{N-1}(-1)^{j+1-i}\left(\begin{array}{c}
m+N \\
j+m+1
\end{array}\right)\left(\begin{array}{l}
j \\
i
\end{array}\right) \\
= & \operatorname{LHS}_{i-1, N-1}^{(m+1)}-\operatorname{LHS}_{i, N-1}^{(m+1)} .
\end{aligned}
$$

Step 3, (induction) : By step 1, it holds that $\operatorname{LHS}_{0, N}^{(m)}=\operatorname{RHS}_{0, N}^{(m)}$ whenever $N \geq 0$, and $m \geq 1$. Moreover, $\operatorname{LHS}_{N, N}^{(m)}=\operatorname{RHS}_{N, N}^{(m)}$ for any $N \geq 0, m \geq 1$. We may thus assume that there exists $i \geq 0$ such that

$$
m \geq 1, N \geq i \quad \Rightarrow \quad \operatorname{LHS}_{i, N}^{(m)}=\operatorname{RHS}_{i, N}^{(m)},
$$

and $N \geq i+1$ such that

$$
m \geq 1 \Rightarrow \operatorname{LHS}_{i+1, N}^{(m)}=\operatorname{RHS}_{i+1, N}^{(m)}
$$


By step 2, this implies that

$$
\begin{aligned}
\operatorname{LHS}_{i+1, N+1}^{(m)} & =\operatorname{LHS}_{i, N}^{(m+1)}-\operatorname{LHS}_{i+1, N}^{(m+1)} \\
& =\operatorname{RHS}_{i, N}^{(m+1)}-\operatorname{RHS}_{i+1, N}^{(m+1)}=\operatorname{RHS}_{i+1, N+1}^{(m)} .
\end{aligned}
$$

By induction over $N \geq i+1$,

$$
m \geq 1, N \geq i+1 \Rightarrow \operatorname{LHS}_{i+1, N}^{(m)}=\operatorname{RHS}_{i+1, N}^{(m)} .
$$

By induction over $i \geq 0$,

$$
m \geq 1, N \geq i \geq 0 \Rightarrow \operatorname{LHS}_{i, N}^{(m)}=\operatorname{RHS}_{i, N}^{(m)} .
$$

Proposition 6.2 implies an analogue of Theorem 3.3:

Proposition 6.4. Let $\left(T_{t}\right)_{t \geq 0}$ be an $m$-concave semigroup with generator $A$. If $y \in D\left(A^{m-1}\right)$, then

$$
\left\|T_{t} y\right\|^{2} \leq \sum_{j=0}^{m-1} \frac{t^{j}}{j !} \alpha_{j}^{A}(y), \quad t \geq 0,
$$

where $\left(\alpha_{j}^{A}\right)_{j=0}^{m-1}$ is given by $(2.5)$.

A significant difference from the $m$-isometric case is that we have no reason to expect the forms $\left(\alpha_{j}^{A}\right)_{j=0}^{m-1}$ to be bounded. Therefore, we obtain no evidence that $m$-concave semigroups are quasicontractive by necessity. On the other hand, from Proposition 6.2, we have that

$$
T_{k}^{*} T_{k} \leq \sum_{j=0}^{m-1}\left(\begin{array}{l}
k \\
j
\end{array}\right) \beta_{j}\left(T_{1}\right) .
$$

Each form $x \mapsto\left\langle\beta_{j}\left(T_{1}\right) x, x\right\rangle$ is bounded on $\mathcal{H}$. Together with the semigroup property, this implies that $\left\|T_{t}\right\|^{2} \lesssim(1+t)^{m-1}$. From (2.9), we therefore obtain:

Proposition 6.5. Let $m \in \mathbb{Z}_{\geq 0}$, and $\left(T_{t}\right)_{t \geq 0}$ be an $m$-concave semigroup with generator $A$. Then

$$
\sigma(A) \subseteq\{z \in \mathbb{C} ; \operatorname{Re} z \leq 0\} .
$$

In particular, $\left(T_{t}\right)_{t \geq 0}$ has a well-defined cogenerator

$$
T=(A+I)(A-I)^{-1} \in \mathcal{L} .
$$

Combining the above result with Proposition 6.1, and Lemma 2.1, one obtains:

Proposition 6.6. A $C_{0}$-semigroup $\left(T_{t}\right)_{t \geq 0}$ is $m$-concave if and only if it possess an m-concave cogenerator.

\section{Acknowledgements}

During the preparation of this work, I have enjoyed interesting conversations with Jonathan Partington, Bartosz Malman, and Mikael Persson Sundqvist. 
I am also indebted to the anonymous referee for carefully reading and commenting on several versions of this manuscript. In particular, the suggestion to include a more thorough discussion on generators led to substantial improvements not only of the presentation, but also of the results.

Open Access. This article is distributed under the terms of the Creative Commons Attribution 4.0 International License (http://creativecommons.org/licenses/by/4. $0 /$ ), which permits unrestricted use, distribution, and reproduction in any medium, provided you give appropriate credit to the original author(s) and the source, provide a link to the Creative Commons license, and indicate if changes were made.

Publisher's Note Springer Nature remains neutral with regard to jurisdictional claims in published maps and institutional affiliations.

\section{References}

[1] Agler, J.: A disconjugacy theorem for Toeplitz operators. Am. J. Math. 112(1), 1-14 (1990)

[2] Agler, J., Stankus, M.: $m$-isometric transformations of Hilbert space I. Integral Equ. Operator Theory 21(4), 383-429 (1995)

[3] Agler, J., Stankus, M.: $m$-isometric transformations of Hilbert space II. Integral Equ. Operator Theory 23(1), 1-48 (1995)

[4] Agler, J., Stankus, M.: $m$-isometric transformations of Hilbert space III. Integral Equ. Operator Theory 24(4), 379-421 (1996)

[5] Bermúdez, T., Bonilla, A., Zaway, H.: $C_{0}$-semigroups of $m$-isometries on Hilbert spaces. J. Math. Anal. Appl. 472(1), 879-893 (2019)

[6] Bermúdez, T., Martinón, A., Müller, V.: $(m, q)$-isometries on metric spaces. J. Oper. Theory 72(2), 313-328 (2014)

[7] El-Fallah, O., Kellay, K., Mashreghi, J., Ransford, T.: A Primer on the Dirichlet Space, Cambridge Tracts in Mathematics, vol. 203. Cambridge University Press, Cambridge (2014)

[8] Engel, K.-J., Nagel, R.: One-Parameter Semigroups for Linear Evolution Equations, Graduate Texts in Mathematics, vol. 194. Springer, New York (2000)

[9] Gallardo-Gutiérrez, E.A., Partington, J.R.: $C_{0}$-semigroups of 2-isometries and Dirichlet spaces. Rev. Mat. Iberoam. 34(3), 1415-1425 (2018)

[10] Helton, H.: Operators with a representation as multiplication by $x$ on a Sobolev space, Hilbert space operators and operator algebras, North-Holland, Amsterdam, 1972. In: Proceedings of an International Conference held at Tihany, 14-18 September 1970, pp. 279-287 (loose errata)

[11] Jacob, B., Partington, J.R., Pott, S., Wynn, A.: $\beta$-admissibility of observation operators for hypercontractive semigroups. J. Evol. Equ. 18(1), 153-170 (2018)

[12] Nikolski, N.K.: Operators, Functions, and Systems: An Easy Reading. Vol. I, Mathematical Surveys and Monographs, vol. 92. American Mathematical Society, Providence (2002)

[13] Olofsson, A.: Operator-valued $n$-harmonic measure in the polydisc. Studia Math. 163(3), 203-216 (2004)

[14] Olofsson, A.: A von Neumann-Wold decomposition of two-isometries. Acta Sci. Math. (Szeged) 70(3-4), 715-726 (2004) 
[15] Richter, S.: A representation theorem for cyclic analytic two-isometries. Trans. Am. Math. Soc. 328(1), 325-349 (1991)

[16] Rosenblum, M., Rovnyak, J.: Hardy Classes and Operator Theory. Oxford Mathematical Monographs. The Clarendon Press, New York (1985)

[17] Rydhe, E.: An Agler-type model theorem for $C_{0}$-semigroups of Hilbert space contractions. J. Lond. Math. Soc. (2) 93(2), 420-438 (2016)

[18] Rydhe, E.: Cyclic $m$-isometries, and Dirichlet type spaces. J. Lond. Math. Soc. 1, 101 (2018). https://doi.org/10.1112/jlms.12199

[19] Shimorin, S.: Wold-type decompositions and wandering subspaces for operators close to isometries. J. Reine Angew. Math. 531, 147-189 (2001)

[20] Sz-Nagy, B., Foias, C., Bercovici, H., Kérchy, L.: Harmonic Analysis of Operators on Hilbert Space, 2nd edn. Springer, New York (2010)

Eskil Rydhe $(\bowtie)$

School of Mathematics

University of Leeds

LS2 9JT Leeds

UK

e-mail: e.rydhe@leeds.ac.uk

Received: September 3, 2018.

Revised: April 23, 2019. 